\title{
MOMENT MAPS AND SYMMETRIC MULTILINEAR FORMS ASSOCIATED WITH SYMPLECTIC CLASSES*
}

\author{
AKITO FUTAKI ${ }^{\dagger}$ AND TOSHIKI MABUCHI ${ }^{\ddagger}$
}

1. Introduction. For a compact connected symplectic $2 n$-dimensional manifold $(M, \omega)$ with a Hamiltonian action of a compact connected Lie group $G$, we have a moment map $\mu: M \rightarrow \mathfrak{g}^{*}$ such that the Hamiltonian functions $\mu_{X}:=\langle\mu, X\rangle$, $X \in \mathfrak{g}$, satisfy the condition

$$
\int_{M} \mu_{X} \omega^{n}=0
$$

where $\mathfrak{g}$ denotes the Lie algebra of $G$. This $\mu$ is uniquely determined by the $G$-action on $M$ as above, and is called the reduced moment map.

Let us first consider the case where $G$ is a $k$-dimensional torus $T=\left(S^{1}\right)^{k}$ with the associated Lie algebra $\mathfrak{t}$. Then the image $\mu(M)$ of the moment map is a compact convex polytope (cf. Atiyah [1], Guillemin and Sternberg [14]). The kernel, denoted by $\mathfrak{t}_{\mathbb{Z}}$, of the exponential map exp : $\mathfrak{t} \rightarrow T$ is called the lattice in $\mathfrak{t}$, and points in the lattice are called integral points. They in turn define the dual lattice $\mathfrak{t}_{\mathbb{Z}}^{*}$ and integral points in $\mathfrak{t}^{*}$. By setting $\mathfrak{t}_{\mathbb{Q}}:=\mathfrak{t}_{\mathbb{Z}} \otimes_{\mathbb{Z}} \mathbb{Q}$ and $\mathfrak{t}_{\mathbb{Q}}^{*}:=\mathfrak{t}_{\mathbb{Z}}^{*} \otimes_{\mathbb{Z}} \mathbb{Q}$, we have rational points in $\mathfrak{t}$ and $\mathfrak{t}^{*}$. A convex polytope in $\mathfrak{t}^{*}$ is said to be integral or rational, according as all vertices are integral points or rational points, respectively. The fixed point set $M^{T}$ of the $T$-action on $M$ sits in the critical point set for $\mu$, and the image $\mu\left(M^{T}\right)$ is a finite subset of $\mathfrak{t}^{*}$. The following proposition, which was originally conjectured by Atiyah [1] in the special case of projective algebraic manifolds, plays a key role in our work:

Proposition A. Assume that $\omega$ represents an integral cohomology class of $M$ modulo torsion. For $G=T=\left(S^{1}\right)^{k}$, let $\mu: M \rightarrow \mathfrak{t}^{*}$ be the reduced moment map. Then $\mu\left(M^{T}\right) \subset \mathfrak{t}_{\mathbb{Q}}^{*}$, and in particular, the convex polytope $\mu(M)$ is rational.

In this paper, by giving a proof of this proposition, we generalize the results in [10] to symplectic cases. Take a general compact Lie group $G$, and let $\mu: M \rightarrow \mathfrak{g}^{*}$ be the reduced moment map as above. We here observe that the underlying differentiable structure of a compact symplectic manifold does not necessarily admit a compatible complex structure (see [13]). For the symplectic class $[\omega]$, just like the $\ell$-th moment for a probability distribution, we define a symmetric multilinear form $\Phi_{\ell}: \otimes^{\ell} \mathfrak{g} \rightarrow \mathbb{R}$ by

$$
\Phi_{\ell}\left(Y_{1}, \cdots, Y_{\ell}\right)=\int_{M} \mu_{Y_{1}} \cdots \mu_{Y_{\ell}} \omega^{n}, \quad Y_{1}, \ldots, Y_{\ell} \in \mathfrak{g}
$$

Let $\mathfrak{g}_{\mathbb{Q}}$ be the set of all $X \in \mathfrak{g}$ such that $m X$ is in the kernel of exp $: \mathfrak{g} \rightarrow G$ for some positive integer $m$. In view of Proposition A, we obtain

*Received February 18, 2002; accepted for publication April 9, 2002. Dedicated to Professor Takushiro Ochiai on his sixtieth birthday.

†Department of Mathematics, Tokyo Institute of Technology, 2-12-1, Oh-Okayama, Meguro, Tokyo, 152-8551 Japan (futaki@math.titech.ac.jp). Partially supported by Institut des Hautes Études Scientifiques.

‡Department of Mathematics, Graduate School of Science, Osaka University, Toyonaka, Osaka, 560-0043 Japan (mabuchi@math.wani.osaka-u.ac.jp). Partially supported by the Korea Institute for Advanced Study. 
Theorem B. (a) For a fixed symplectic class, the multilinear form $\Phi_{\ell}$ is independent of the choice of $\omega$ in the class.

(b) Assume that $X \in \mathfrak{g}_{\mathbb{Q}}$. If $\omega$ represents an integral cohomology class of $M$ modulo torsion, then $\Phi_{\ell}(X, X, \ldots, X)$ is a rational number.

Now, by changing notations, we consider the case where $M$ is a compact complex connected manifold on which a complex Lie group $H$ specified later will act in place of $G$. We further assume that $M$ admits a symplectic form $\omega_{0}$ of type $(1,1)$, namely a pseudo-Kähler form. Let $\mathcal{S}$ denote the set of all symplectic forms on $M$ expressible as

$$
\omega_{\varphi}:=\omega_{0}+(\sqrt{-1} / 2 \pi) \partial \bar{\partial} \varphi
$$

for some real-valued smooth functions $\varphi \in C^{\infty}(M)_{\mathbb{R}}$ on $M$. By $T^{1,0} M$, we denote the holomorphic tangent bundle of $M$, and let $\operatorname{Aut}(M)$ be the group of all holomorphic automorphisms of $M$. Then the linear map

$$
\phi: H^{0}\left(M, \mathcal{O}\left(T^{1,0} M\right)\right) \rightarrow H^{1}(M, \mathcal{O}), \quad \xi \mapsto \phi(\xi):=[i(\xi) \omega],
$$

is independent of the choice of $\omega$ in $\mathcal{S}$. The kernel $\mathfrak{h}:=\operatorname{Ker} \phi$ is called the space of the Hamiltonian holomorphic vector fields on $M$, and will be studied in Theorem 6.1 which generalizes a theorem of Fujiki (cf. Remark 6.2). Here, $\mathfrak{h}$ is not necessarily a complexification of a compactly embedded Lie subalgebra. As in the case of $\Phi_{\ell}: \otimes^{\ell} \mathfrak{g} \rightarrow \mathbb{R}$ above, using the same notation $\Phi_{\ell}$ by abuse of terminology, we can similarly define a symmetric multilinear form $\Phi_{\ell}: \otimes^{\ell} \mathfrak{h} \rightarrow \mathbb{C}$ which depends only on the class $\mathcal{S}$ (cf. Theorem 6.3). Let $H$ be the complex connected Lie subgroup of $\operatorname{Aut}(M)$ associated to the complex Lie subalgebra $\mathfrak{h}$ of $H^{0}\left(M, \mathcal{O}\left(T^{1,0} M\right)\right)$. Then, as an analogue of the Bando-Calabi-Futaki character in Kähler cases, we obtain a Lie algebra character (cf. (20) in Section 6)

$$
\mathcal{F}_{\mathcal{S}}: \mathfrak{h} \rightarrow \mathbb{C}
$$

which is an obstruction to the existence of a symplectic form in $\mathcal{S}$ with constant scalar curvature. Choose a maximal compact subgroup $K$ of $H$, and let $\mathfrak{k}$ be the associated Lie subalgebra of $\mathfrak{h}$, and $\mathfrak{k}_{\mathbb{C}}:=\mathfrak{k}+\sqrt{-1} \mathfrak{k}$ the complexification of $\mathfrak{k}$ in $\mathfrak{h}$. Again as in Kähler cases, the symmetric bilinear form $\Phi_{2}: \otimes^{2} \mathfrak{h} \rightarrow \mathbb{C}$ allows us to obtain an analogue of an extremal Kähler vector field (cf. [10]) as follows:

THEOREM C. If the identity component of the center of $K$ fixes some element of $\mathcal{S}$, then there exists $\xi_{\mathcal{S}}$ in the center of $\mathfrak{k}$, uniquely determined by the choice of $K$, such that

$$
\mathcal{F}_{\mathcal{S}}(\zeta)=\Phi_{2}\left(\zeta, \xi_{\mathcal{S}}\right), \quad \text { for all } \zeta \in \mathfrak{k}_{\mathbb{C}}
$$

Since $K$ is unique up to conjugacy in $H$, so is $\xi_{\mathcal{S}}$ in $\mathfrak{h}$. If the symplectic class of $\mathcal{S}$ is integral modulo torsion, then $\exp \left(\nu \xi_{\mathcal{S}}\right)=1$ for some positive integer $\nu$.

To each symplectic form $\omega \in \mathcal{S}$, we associate a vector filed $\tau_{\omega}:=\operatorname{grad}_{\omega}^{\mathbb{C}} \sigma(\omega)$ (see Section 6), where $\sigma(\omega)$ is the scalar curvature of $\omega$ (cf. Appendix). Then $\omega$ is said to be extremal or strictly extremal, according as $\tau_{\omega}$ is holomorphic (i.e., $\tau_{\omega} \in \mathfrak{h}$ ) or the one-parameter group $\left\{\exp \left(t \tau_{\omega}\right) ; t \in \mathbb{R}\right\}$ sits in a compact subgroup of $H$, respectively. Now, let $\omega$ be an element of $\mathcal{S}$ fixed by the identity component of the center of $K$ above. Then 
Corollary D. (a) If $\omega$ is strictly extremal, then $\tau_{\omega}$ coincides with $\xi_{\mathcal{S}}$ above up to conjugacy in $\mathfrak{h}$, and in particular, both $\max _{M} \sigma(\omega)$ and $\min _{M} \sigma(\omega)$ are rational numbers.

(b) Assume that $\omega$ is extremal. Then $\omega$ is strictly extremal if, for instance, $H$ is a reductive algebraic group or $\omega$ is a Kähler form.

2. Preliminaries. In this paper, with the only exception in Appendix, all symplecic forms are assumed to be real. Furthermore, $M$ is always a compact connected symplectic manifold of dimension $2 n$ with symplectic class $[\omega]$. (In this section, the cohomology class $\left[\omega_{0}\right]$ is assumed to be integral modulo torsion, where the terminology "modulo torsion" is often omitted for simplicity:) Take a complex line bundle $L$ with $c_{1}(L)=[\omega]$ and denote by $P$ the principal $S^{1}$-bundle associated with $L$. Assume that we have a Hamiltonian action of the $k$-dimensional torus $T=\left(S^{1}\right)^{k}$ on $M$, where $T$ is regarded as the only maximal compact subgroup of $\left(\mathbb{C}^{*}\right)^{k}$. In particular, the action of $T$ on $M$ is symplectic, i.e., it preserves the symplectic form $\omega$. Identify $\mathfrak{t}$ with $\mathbb{R}^{k}$ and the lattice in $\mathfrak{t}$ with $\mathbb{Z}^{k}$ sitting in $\mathbb{R}^{k}$. Then the the exponential map exp : $\mathfrak{t} \rightarrow T$ is written as

$$
\left(x_{1}, x_{2}, \ldots, x_{k}\right) \mapsto\left(e^{2 \pi \sqrt{-1} x_{1}}, e^{2 \pi \sqrt{-1} x_{2}}, \ldots, e^{2 \pi \sqrt{-1} x_{k}}\right) .
$$

Each $X \in \mathfrak{t}$ induces a symplectic vector field, denoted also by $X$, on $M$. We further have a moment map $\mu: M \rightarrow \mathfrak{t}^{*}$ which is, in general, unique only up to translation. Then

$$
d<\mu, X>=i(X) \omega
$$

and $\mu$ is equivariant. The function $\mu_{X}:=\langle\mu, X\rangle$ is $T$-invariant, and is called the Hamiltonian function for $X \in \mathfrak{t}$. Given a Hamiltonian $T$-action on $M$, if $\mu: M \rightarrow \mathfrak{t}$ is a reduced moment map, it is uniquely determined by the action.

Suppose for a moment that the symplectic action is given by a general compact Lie group $G$ with Lie algebra $\mathfrak{g}$ instead of the torus $T$ (this is just to make clear the motivation of the construction (2.2) below). Let $L$ be a complex line bundle with $c_{1}(L)=[\omega]$. Take a connection $\nabla$ of $L$ such that its curvature $R(\nabla)$ is equal to $-2 \pi \sqrt{-1} \omega$. Now we will see that a moment map gives rise to a Lie algebra homomorphism $\mathfrak{g} \rightarrow \mathfrak{X}(L)$ where $\mathfrak{X}(L)$ denotes the Lie algebra of all vector fields on $L$. It is also possible to replace $L$ by the associated principal bundle $P$. Namely a moment map gives rise to a Lie algebra homomorphism $\mathfrak{g} \rightarrow \mathfrak{X}(P)$. We will explain this in the case of $P$; the case of $L$ is quite similar. For any $X \in \mathfrak{g}$ considered as a vector field on $M$, there is a horizontal lift $X^{h}$ on $P$ with respect to the connecion induced from $\nabla$. Locally this is expressed as follows. Let $U$ be an open set, and let $\left.P\right|_{U} \cong U \times S^{1}$ and $\left.L\right|_{U} \cong U \times \mathbb{C}$ be local trivializations. Let $\theta_{U}$ be the connection form on $M$ with respect to this trivialization. Then the associated connection form in $P$ is expressible as

$$
\tilde{\theta}=\frac{d z}{z}+\theta_{U}
$$

and the horizontal lift of $X$ is given by

$$
X^{h}=-\theta_{U}(X) z \frac{\partial}{\partial z}+X
$$


where $z$ denotes the fiber coordinate of $\left.L\right|_{U} \cong U \times \mathbb{C}$ and $d z / z$ is the Maurer-Cartan form of $S^{1} \subset \mathbb{C}$. This lift gives a map $\mathfrak{g} \rightarrow \mathfrak{X}(P)$ which is however not a Lie algebra homomorphism. We add $2 \pi \mu_{X} \sqrt{-1} z \partial / \partial z$ to define $\mathfrak{g} \rightarrow \mathfrak{X}(P)$ by

$$
X \mapsto X^{\sharp}:=2 \pi \mu_{X} \sqrt{-1} z \frac{\partial}{\partial z}+X^{h},
$$

which is easily seen to be a Lie algebra homomorphism. Now we again return to the case of symplectic torus action. To fix our notation, we give a geometric proof to the following fact (see Ono [21; Lemma (4.8)]):

Proposition 2.1. The action of $T=\left(S^{1}\right)^{k}$ lifts to bundle actions on $P$ and $L$.

Proof. Clearly we have only to show this for $P$. Let $X$ be a generator of the lattice in $\mathfrak{t}$, and $\left\{\psi_{t}\right\}_{t \in \mathbb{R}}$ be the one-parameter group generated by $X^{\sharp}$. Since $\psi_{t}$ covers the $S^{1}$ action on $M$ generated by $X$, we see that $\psi_{1}$ defines a gauge transformation (denoted also by $\psi_{1}$ by abuse of terminology) of $P$, i.e., a smooth map from $M$ to $S^{1}$.

We claim that this gauge transformation is homotopic to a constant map. To see this, note that the set of homotopy classes of maps from $M$ to $S^{1}$ is isomorphic to $H^{1}(M ; \mathbb{Z})$ by assigning $\phi: M \rightarrow S^{1}$ to $\phi^{*} \alpha \in H^{1}(M ; \mathbb{Z})$, where $\alpha$ denotes the generator of $H^{1}\left(S^{1} ; \mathbb{Z}\right)$. Thus it suffices to see $\psi_{1}^{*} \alpha=0$. Since $\mu_{X}$ is a perfect Morse function,

$$
H^{1}(M ; \mathbb{Z}) \cong H_{2 n-1}(M ; \mathbb{Z}) \cong \oplus_{i} H_{2 n-1-2 \lambda_{i}}\left(F_{i} ; \mathbb{Z}\right)
$$

where $F_{i}$ are connected componets of the set of critical points of $\mu_{X}$, and $2 \lambda_{i}$ is the index of $F_{i}$. Let $2 d_{i}$ be the dimension of $F_{i}$. Since $2 n-2 \lambda_{i} \geq 2 d_{i}$, the only non-zero contribution of the last term is

$$
\oplus_{i} H_{2 d_{i}-1}\left(F_{i} ; \mathbb{Z}\right) \cong \oplus_{i} H^{1}\left(F_{i} ; \mathbb{Z}\right)
$$

Thus it suffices to see $\left.\psi_{1}\right|_{F_{i}}: F_{i}(\hookrightarrow M) \rightarrow S^{1}$ is homotopic to a constant map. But this is the case, since $X$ vanishes and $\mu_{X}$ is constant along $F_{i}$.

It follows that $\psi_{1}$ lifts to a map $\widetilde{\psi}: M \rightarrow \mathbb{R}$. Put $\widetilde{X}:=X^{\sharp}-\widetilde{\psi} \sqrt{-1} z \partial / \partial z$. Noting that $\psi_{1}$ and $\widetilde{\psi}$ are $S^{1}$-invariant under the $S^{1}$-action on $M$, we see that $\widetilde{X}$ generates an $S^{1}$-action on $L$ covering that on $M$.

REMARK 2.2. As the proof shows, the lifting in Proposition 2.1 is not unique. In fact, the choice of $\widetilde{\psi}$ is determined up to modulo $2 \pi \mathbb{Z}$. We will see in Theorem 2.4 that a choice of lifting reflects on a translation of the image of moment maps (see also the example given at the end of this section). We may also interpret this as the exact sequence over $\mathbb{Z}$ given in Fujiki [7; Lemma 3.1].

REMARK 2.3. Let $M$ be a compact complex manifold with a pseudo-Kähler form $\omega$ (cf. Section 6) in an integral class, and take a holomorphic line bundle $L$ such that $c_{1}(L)=[\omega]$. Choose a Hermitian connection $\theta$ on $L$ such that $d \theta=-2 \pi \sqrt{-1} \omega$. Then for a holomorphic vector field $\xi$ on $M$ which generates a Hamiltonian $S^{1}$-action on $M$, the associated lifting $\xi^{\sharp}$ on $L$ induced by $(2.2)$ is holomorphic on $L$, since $\bar{\partial} \mu_{X}=i(\xi) \omega$ and $\bar{\partial}(\theta(X))=-2 \pi \sqrt{-1} i(\xi) \omega$, where $X:=\xi+\bar{\xi}$ and $X^{\sharp}=\xi^{\sharp}+\bar{\xi}^{\sharp}$. In this case, Proposition 2.1 is written as follows (see also [15]):

Let $M$ be a compact complex manifold with a pseudo-Kähler form $\omega$ in an integral cohomology class, and let $P$ and $L$ be as above. Then a holomorphic $\left(\mathbb{C}^{*}\right)^{k}$-action on $M$ extending a Hamiltonian torus action lifts to a holomorphic bundle action on $L$. 
Next, coming back to general symplectic situations, we recall a result of BerlineVergne [3]. Let $G$ and $G^{\prime}$ be compact Lie groups with Lie algebras $\mathfrak{g}$ and $\mathfrak{g}^{\prime}$, and let $P \rightarrow M$ be a principal $G$-bundle endowed with a left $G^{\prime}$-action on $P$ which covers a $G^{\prime}$-action on $M$ and commutes with the $G$-action on $P$. Assume $\operatorname{dim}_{\mathbb{R}} M=2 n$. For integers $j \geq 0$, put

$$
I^{j}(G)=S^{j}\left(\mathfrak{g}^{*}\right)^{G}, \quad \text { and } \quad I^{j}\left(G^{\prime}\right)=S^{j}\left(\mathfrak{g}^{\prime *}\right)^{G},
$$

i.e., the former (resp. the latter) is the set of all $G$-invariant (resp. $G^{\prime}$-invariant) symmetric polynomials of degree $j$ with coefficients in $\mathbb{C}$ and with variables in $\mathfrak{g}^{*}$ $\left(\right.$ resp. $\left.\mathfrak{g}^{\prime *}\right)$. Choose a $G^{\prime}$-invariant connection $\tilde{\theta}$ of $P \rightarrow M$. For any $\phi \in I^{j}(G)$, we define

$$
f_{\phi}(X)=\int_{M} \phi(\tilde{\theta}(X)+\Theta)
$$

where $X \in \mathfrak{g}^{\prime}$ is regarded as a vector field on $P$, and $\Theta$ denotes the curvature form of $\tilde{\theta}$. Then $f_{\phi}$ turns out to be independent of the choice of $\theta$, and defines an element of $I^{j}\left(G^{\prime}\right)$. We apply this result to our symplectic situation. By setting $G=S^{1}$ and $G^{\prime}=T=\left(S^{1}\right)^{k}$, let $(M, \omega)$ be a compact symplectic manifold with a Hamiltonian $T$-action. Let $L$ over $(M, \omega)$ be a complex line bundle with $c_{1}(L)=[\omega]$. Then by Proposition 2.1, the $T$-action on $M$ lifts to a bundle action on $L$. Choose such a lifting. For the associated $S^{1}$-bundle $P$, we consider the invariant polynomial $c_{1}^{n+1} \in I^{n+1}\left(S^{1}\right)$ defined by

$$
c_{1}^{n+1}(x):=\left(\frac{\sqrt{-1} x}{2 \pi}\right)^{n+1} .
$$

Then $f_{c_{1}^{n+1}}^{L} \in I^{n+1}(T)$ is written as

$$
\begin{aligned}
f_{c_{1}^{n+1}}^{L}(X) & =\left(\frac{\sqrt{-1}}{2 \pi}\right)^{n+1} \int_{M}(\tilde{\theta}(X)+\Theta)^{n+1} \\
& =(n+1) \int_{M} \frac{\sqrt{-1}}{2 \pi} \tilde{\theta}(X) \omega^{n}
\end{aligned}
$$

where $\theta$ is a $T$-invariant connection with $\Theta=d \theta=-2 \pi \sqrt{-1} \omega$ and $\widetilde{\theta}$ is the connection form in $P$ which of course is locally equal to the Maurer-Cartan form plus $\theta$. Moreover, $X$ denotes the vector field (denoted by $\widetilde{X}$ in the proof of Proposition 2.1) on $P$ corresponding to $X \in \mathfrak{t}$. Let $X_{1}, \cdots, X_{k}$ be the generators of the lattice of $\mathfrak{t}$. We set

$$
d_{i}=-(n+1)^{-1} f_{c_{1}^{n+1}}^{L}\left(X_{i}\right)
$$

It should be noted that $d_{1}, \cdots, d_{k}$ depend on the lifting to $L$ of the $T$-action on $M$.

LEMMA 2.4. Let $\mu_{X_{i}}$ be such that $\mu_{X_{i}}=\{2 \pi \sqrt{-1}\}^{-1} \widetilde{\theta}\left(X_{i}\right)$. This amounts to normalizing $\mu_{X_{i}}$ by

$$
\int_{M} \mu_{X_{i}} \omega^{n}=d_{i}
$$

Proof. Since $\tilde{\theta}$ is $T$-invariant, $L_{X} \widetilde{\theta}=0$. But $L_{X} \widetilde{\theta}=d(\widetilde{\theta}(X))+i(X) \Theta$ and hence

$$
i(X) \omega=d\left(\{2 \pi \sqrt{-1}\}^{-1} \tilde{\theta}(X)\right) .
$$


Now (2.7) follows from (2.5) and (2.6).

We now consider the integrality of the image of the moment map. This is wellknown for toric varieties and Delzant spaces [4]. More generally, Guillemin and Sternberg show in [14] that the integrality holds for prequantizable Hamiltonian torus actions. The following theorem is proved essentially by showing that such a prequantization is realized by the normalization (2.7) of the Hamiltonian functions:

THEOREM 2.5. (a) Let $M$ be a compact connected symplectic manifold with integral symplectic class $[\omega]$. Suppose that $M$ admits a Hamiltonian action of $T=$ $\left(S^{1}\right)^{k}$. Let $L$ be a complex line bundle with $c_{1}(L)=[\omega]$, and choose a lifting of the $T$-action on $M$ to a bundle action on $L$. Normalize the Hamiltonian functions $\mu_{X_{i}}, 1 \leq i \leq k$, such that (2.7) is satisfied with respect to the lifting. Then each critical value of $\mu_{X_{i}}$ is the integer obtained as the common weight of the isotropy action of $S^{1}$ generated by $X_{i}$ on the fibers of $L$ over the associated critical points, and the image of the moment map $\mu=\left(\mu_{X_{1}}, \ldots, \mu_{X_{k}}\right)$ is an integral convex polytope.

(b) Moreover, for any translation of the image of the moment map to an integral convex polytope, there exists a lifting of the T-action to a bundle action on $L$ such that the translated Hamiltonian functions satisfies (2.7) with respect to the lifting.

Proof. (a) For a generator $X_{i} \in \mathfrak{t}$, the associated vector field on $P$ induced from the lifting of the $T^{k}$-action will be denoted by the same letter $X_{i}$. By the previous lemma, under the normalization (2.7), we have $\mu_{X_{i}}=(2 \pi \sqrt{-1})^{-1} \tilde{\theta}\left(X_{i}\right)$. For a critical point $p \in M$ of $\mu_{X_{i}}$,

$$
\mu_{X_{i}}(p)=\frac{1}{2 \pi \sqrt{-1}} \tilde{\theta}\left(X_{i}\right)_{p}=\frac{1}{2 \pi \sqrt{-1}} \frac{d z}{z}\left(X_{i}\right)_{p}
$$

is the weight of the isotropy action of $S^{1}$ generated by $X_{i}$ on the fiber $L_{p}$ over $p$. Thus the critical values of $\mu_{X_{i}}$ are integers. Now for a vertex $v=\left(v_{1}, \cdots, v_{k}\right) \in \mathfrak{t}^{*}$ of the image of the moment map, $v_{i}$ is a critical value of $\mu_{X_{i}}$, and hence $v$ is an integral point.

(b) To prove the last statement, recall the proof of Proposition 2.1. By Remark 2.2 $\widetilde{\psi}$ is unique up to modulo $2 \pi \mathbb{Z}$. Let $p$ be a critical point of $\mu_{X_{i}}$. Then for the $S^{1}$ action on $M$ generated by $X_{i}$, any integer can be taken as the weight of the isotropy $S^{1}$-action on $L_{p}$ for some lifting to $L$ of the $S^{1}$-action. Hence any translation to an integral polytope is obtained by choosing a some another lifting of the $T$-action to $L$.

REMARK 2.6. Theorem 2.5 implies, for each $\mu_{X_{i}}$, that if one critical value is an integer then all other critical values are integers.

Let us look at an example. Take $M$ to be the 1-dimensional complex projective space $\mathbb{P}=\mathbb{P}^{1}(\mathbb{C})$, and $S^{1}$ to be in the center of $\operatorname{PGL}(2, \mathbb{C})$. Let $\left(z_{0}, z_{1}\right)$ be the homogeneous coordinates and $u=z_{1} / z_{0}$ be the inhomogeneous coordinate on the open set $\left\{z_{0} \neq 0\right\}$. Then the $S^{1}$-action is given by $u \mapsto e^{2 \pi i t} u$. The Kähler form

$$
\omega_{F S}:=\frac{\sqrt{-1}}{\pi} \frac{d u \wedge d \bar{u}}{\left(1+|u|^{2}\right)^{2}}
$$

represents the first Chern class of $M$, i.e. $c_{1}\left(T^{1,0} \mathbb{P}\right)=\left[\omega_{F S}\right]$. For the generator

$$
X=2 \pi \sqrt{-1} u \frac{\partial}{\partial u}-2 \pi \sqrt{-1} \bar{u} \frac{\partial}{\partial \bar{u}}
$$


of the $S^{1}$-action we have $i(X) \omega_{F S}=d\left\{\left(1-|u|^{2}\right) /\left(1+|u|^{2}\right)\right\}$, and thus

$$
\mu\left(z_{0}: z_{1}\right)=\frac{\left|z_{0}\right|^{2}-\left|z_{1}\right|^{2}}{\left|z_{0}\right|^{2}+\left|z_{1}\right|^{2}}
$$

is the moment map, whose image is clearly an interval with integral end points. However, $\omega:=\omega_{F S} / 2$ is still an integral class which is the Chern class of the hyperplane bundle $L$, i.e. the square root of $T^{1,0} \mathbb{P}$. The moment map for $\omega$ is $\mu / 2$ whose image is the interval $[-1 / 2,1 / 2]$. We now see what was done in the proof of Proposition 2.1. The connections $\theta, \widetilde{\theta}$ and $X^{\sharp}$ are respectively given as follows:

$$
\theta=\frac{-1}{2}\left(\frac{\bar{u} d u}{1+|u|^{2}}-\frac{u d \bar{u}}{1+|u|^{2}}\right)
$$

where $\theta$ is the Hermitian connection of the standard metric of $L$, and on the associated $S^{1}$-bundle $P$, we have $\widetilde{\theta}=\sqrt{-1} d \lambda+\theta$ for the fiber coordinate $z=e^{\sqrt{-1} \lambda}$ of $P$. Moreover,

$$
X^{\sharp}=(2 \pi \mu / 2+\sqrt{-1} \theta(X)) \frac{\partial}{\partial \lambda}+X=\pi \frac{\partial}{\partial \lambda}+X .
$$

From this, it follows that $\psi_{1}=\pi$. Choose $\widetilde{\psi}=(-2 m+1) \pi$ for an integer $m$. Then $\widetilde{X}=2 m \pi \partial / \partial \lambda+X$. The resulting lifting gives the moment map

$$
u \mapsto \frac{1}{2 \pi \sqrt{-1}} \widetilde{\theta}(\widetilde{X})=m-\frac{|u|^{2}}{1+|u|^{2}},
$$

whose image is the interval $[m-1, m]$.

3. The localization formula. In this section, let $k=1$, i.e., we consider a compact symplectic manifold $(M, \omega)$ with a Hamiltonian action of $T=S^{1}$, where $\mu: M \rightarrow \mathfrak{t}^{*}$ is the associated reduced moment map. For $0 \neq X \in \mathfrak{t}$, the zero set $N$ of $Y$ is written as a finite disjoint union

$$
N=\bigcup_{\gamma \in \Gamma} N_{\gamma}
$$

of the connected components $N_{\gamma}$. Then $N$ is a symplectic submanifold of $M$, and its normal bundle $E$ is a symplectic vector bundle which naturally becomes a complex vector bundle. The restriction to $E$ of minus Lie derivative $-L_{X}$ is a complex endomorphism, which we denote by $L X$. In fact, when we write $\omega$ as the standard symplectic form on $\mathbb{C}^{n}$ using the Darboux-Weinstein theorem, the type $(1,0)$ part of $X$ can be written locally as a holomorphic vector field

$$
\xi=2 \pi \sqrt{-1} \sum_{i} \xi^{i} \frac{\partial}{\partial z^{i}}
$$

on an open set of $\mathbb{C}^{n}$. Thus for a normal vector $\partial / \partial z^{\lambda}$,

$$
(L X) \frac{\partial}{\partial z^{\lambda}}=-2 \pi \sqrt{-1}\left[\xi+\bar{\xi}, \partial / \partial z^{\lambda}\right]=2 \pi \sqrt{-1} \sum_{\tau} \frac{\partial \xi^{\tau}}{\partial z^{\lambda}} \frac{\partial}{\partial z^{\tau}} \bmod T N
$$


where $z^{\tau}$ runs over coordinates in the normal direction to $N$. Let $\ell$ be a positive integer and $C$ an arbitrary real constant. By the well-known residue formula (see for exponential case [5; Addendum]), we obtain

$$
\int_{M}\left(\mu_{X}+C+\omega\right)^{n+\ell}=\sum_{\gamma \in \Gamma} \int_{N_{\gamma}} \frac{\left(\mu_{X}+C+\omega\right)^{n+\ell}}{\operatorname{det}\left\{\left(L X+\Theta_{E}\right) /(2 \pi \sqrt{-1})\right\}}
$$

where $\Theta_{E}$ denotes the curvature of the conncetion for the normal bundle $E$ of $N$. Since $X$ generates an $S^{1}$-action, all eigenvalues of $L X / 2 \pi \sqrt{-1}$ are real constants, while the function $\mu_{X}:=\langle\mu, X\rangle$ are constant on each $N_{\gamma}$. It then follows that

$$
\delta_{\gamma}(X, C, \ell):=\frac{n ! \ell !}{(n+\ell) !} \int_{N_{\gamma}} \frac{\left(\mu_{X}+C+\omega\right)^{n+\ell}}{\operatorname{det}\left\{\left(L X+\Theta_{E}\right) /(2 \pi \sqrt{-1})\right\}}, \quad \gamma \in \Gamma,
$$

are real constants. Then by (3.1), we obtain the following localization formula:

Proposition 3.1. $\int_{M}\left(\mu_{X}+C\right)^{\ell} \omega^{n}=\sum_{\gamma \in \Gamma} \delta_{\gamma}(X, C, \ell)$.

4. Proof of Proposition A. In this section, we give some consequeces of Theorem 2.5. We shall first prove Proposition A. Let $M, \omega, G$ and $\mu$ be as in Proposition $\mathrm{A}$ in the introduction.

Proof of Proposition A. For $L$ as in (a) of Theorem 2.5, we choose a lifting of the action of $T=\left(S^{1}\right)^{k}$ to $L$. This determines a normalization of the moment map $\nu: M \rightarrow \mathfrak{t}^{*}$ whose image is an integral convex polytope. Apply Proposition 3.1 to $\ell=1$ and $C:=\nu_{X}-\mu_{X}$ with $X=X_{i}$, where $X_{1}, \ldots, X_{k}$ are as in Theorem 2.5. By $X_{i} \in \mathfrak{t}_{\mathbb{Z}}$, all eigenvalues of $L X_{i} / 2 \pi \sqrt{-1}$ are integers, and corresponding to the eigenspace decomposition, $E$ splits into a direct sum of complex vector bundles. Moreover, by Theorem 2.5, the function $\nu_{X}:=\langle\nu, X\rangle=\mu_{X}+C$ takes integer values on $N$. Therefore

$$
\delta_{\gamma}\left(X_{i}, C, 1\right) \in \mathbb{Q}, \quad \gamma \in \Gamma
$$

because $[\omega]$ is an integral class. Then by Proposition 3.1, $\int_{M} \nu_{X_{i}} \omega^{n}=\int_{M}\left(\mu_{X}+\right.$ C) $\omega^{n} \in \mathbb{Q}$, and hence by Theorem 2.5, we conclude that $\mu_{X_{i}}=\nu_{X_{i}}$ $\left\{\int_{M} \omega^{n}\right\}^{-1} \int_{M} \nu_{X_{i}} \omega^{n}$ takes values in $\mathbb{Q}$ on $M^{T}$, as required.

REMARK 4.1. This proof shows that $d_{i}, i=1,2, \ldots, k$, in (2.6) are rational numbers. Later, we see that this rationality follows also from the viewpoints of equivariant cohomologies.

We now study Fano cases. A compact complex manifold is called a Fano manifold if the first Chern class $c_{1}(M)_{\mathbb{R}}$ is represented by a positive closed $(1,1)$-form $\omega$, so that we may regard $c_{1}(M)_{\mathbb{R}}$ as a Kähler class. The Ricci form $\operatorname{Ric}(\omega)=$ $(\sqrt{-1} / 2 \pi) \bar{\partial} \partial \log \omega^{n}$ also represents $c_{1}(M)_{\mathbb{R}}$ by the Chern-Weil theory. Thus there exists a unique smooth function $F$ on $M$, satisfying $\int_{M} e^{F} \omega^{n}=\int_{M} \omega^{n}$, such that

$$
\operatorname{Ric}(\omega)-\omega=(\sqrt{-1} / 2 \pi) \partial \bar{\partial} F \text {. }
$$

Suppose that $T=\left(S^{1}\right)^{k}$ acts on $M$ as isometries. Since $M$ is a Fano manifold, it is well-known that such an action is holomorphic and Hamitonian (see [18]). The action naturally lifts to the anti-canonical bundle $K_{M}^{-1}$. For each $X \in \mathfrak{t}$, we consider the invariant $f_{c_{1}^{n+1}}(X)$ with respect to the natural lifting to $K_{M}^{-1}$ of the $T$-action on $M$. 
Proposition 4.2. For a Hamiltonian function $\mu_{X}$ for $X \in \mathfrak{t}$ with respect to $\omega$, which is unique up to a constant, the normalization

$$
\int_{M} \mu_{X} \omega^{n}=-(n+1)^{-1} f_{c_{1}^{n+1}}(X)
$$

is equivalent to

$$
\int_{M} \mu_{X} e^{F} \omega^{n}=0
$$

Proof. By the Calabi-Yau theorem there exists a Kähler form $h$ in the Kähler class $c_{1}(M)$ such that $\operatorname{Ric}(h)=\omega$. Then $h^{n}$ defines a Hermitian metric on $K_{M}^{-1}$, and let $\theta$ be the associated Hermitian connection and $\Theta$ its curvature form. Obviously $(\sqrt{-1} / 2 \pi) \Theta=\operatorname{Ric}(h)=\omega$. As usual, we denote by $X$ the vector field on $K_{M}^{-1}$ induced

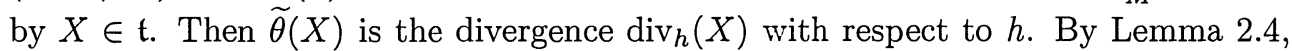
the equality (4.1) holds if and only if

$$
\mu_{X}=(2 \pi \sqrt{-1})^{-1} \tilde{\theta}(X)=(2 \pi \sqrt{-1})^{-1} \operatorname{div}_{h}(X)
$$

which in turn is equivalent to

$$
\int_{M} \mu_{X} h^{n}=\int_{M} \operatorname{div}_{h}(X) h^{n}=0
$$

But, since $\operatorname{Ric}(h)=\omega$, we have $\operatorname{Ric}(h)=\operatorname{Ric}(\omega)-(\sqrt{-1} / 2 \pi) \partial \bar{\partial} F$, i.e., $h^{n}=e^{F} \omega^{n}$. Hence, (4.3) is equivalent to (4.2). This completes the proof.

Corollary 4.3. Let $M$ be a Fano manifold with a Kähler form $\omega$ in the class $c_{1}(M)_{\mathbb{R}}$. Suppose that $T=\left(S^{1}\right)^{k}$ acts on $(M, \omega)$ in a Hamiltonian way with generators $X_{1}, \cdots, X_{k}$ for the lattice in $\mathfrak{t}$. If we normalize the Hamiltonian functions $\mu_{X_{i}}$ by

$$
\int_{M} \mu_{X_{i}} e^{F} \omega^{n}=0
$$

for the function $F$ as above, then the image of the moment map is an integral convex polytope. In particular, if $M$ admits a Kähler-Einstein form $\omega$, then the image of the reduced moment map is an integral convex polytope.

Proof. The first assertion follows from Theorem 2.5 and Proposition 4.2. For Kähler-Einstein forms $\omega$, we have $\operatorname{Ric}(\omega)=\omega$ and hence $F$ is a constant function, and therefore the second assertion follows.

REMARK 4.4. (a) In view of a theorem of Futaki and Morita [11], $f_{c_{1}^{n+1}}(X)$ is an obstruction to the existence of Kähler-Einstein metrics (cf. [8]). In particular

$$
d_{i}=-(n+1)^{-1} f_{c_{1}^{n+1}}\left(X_{i}\right)=0
$$

for Kähler-Einstein forms $\omega$. Thus, without using Proposition 4.2, we have the second assertion of Corollary 4.3 immediately from Theorem 2.5.

(b) Even without Theorem 2.5 and Proposition 4.2, we obtain the first assertion of Corollary 4.3 as follows: For a Kähler form $\omega$ in the class $c_{1}(M)_{\mathbb{R}}$ on a Fano manifold $M$, using the same notation as in Section 5 , we can write every holomorphic vector 
field $\xi$ on $M$ as $\xi=\operatorname{grad}_{\omega}^{\mathbb{C}} u$ for some complex-valued smooth function $u$ on $M$ satisfying the equation

$$
\left.\Delta_{\omega} u+\sum_{\alpha, \beta} g^{\alpha \bar{\beta}} \frac{\partial F}{\partial z^{\alpha}} \frac{\partial u}{\partial \bar{z}^{\beta}}+u=0, \quad \text { (cf. }[9 ; 2.4]\right)
$$

where $\Delta_{\omega}$ denotes the complex Laplacian $\Sigma_{\alpha, \beta} g^{\alpha \bar{\beta}}\left(\partial^{2} / \partial z^{\alpha} \partial \bar{z}^{\beta}\right)$. Such a function $u$ obviously satisfies

$$
\int_{M} u e^{F} \omega^{n}=0
$$

For the generators $X_{1}, X_{2}, \ldots, X_{k}$ of the lattice in $\mathfrak{t}$ in Corollary 4.3 , let $\xi_{i}$ be the associated holomorphic vector field such that $X_{i}=\xi_{i}+\bar{\xi}_{i}, i=1,2, \ldots, k$. Denote by $u_{i}$ the function in $V$ corresponding to $\xi_{i}$, so that we have $\xi_{i}=\operatorname{grad}_{\omega}^{\mathbb{C}} u_{i}$. Then

$$
\mu_{X_{i}}=u_{i}, \quad i=1,2, \ldots, k .
$$

Let $p \in M$ be a critical point of $\mu_{X_{i}}$. Since $\operatorname{grad}_{\omega}^{\mathbb{C}} u_{i}=0$ at $p$, by using the equation above, we obtain

$$
-\mu_{X_{i}}(p)=-u_{i}(p)=\left(\Delta_{\omega} u_{i}\right)(p)
$$

But the right-hand side is an integer because it is the sum of the weigts of the $S^{1}$ action on the normal bundle of the component containing $p$ of the fixed point set, or equivalently the weight of the action on $\left(K_{M}^{-1}\right)_{p}$. Thus $\mu_{X_{i}}$ takes values in $\mathbb{Z}$ on the critical point set. Therefore, the image of the moment map is a convex polytope with integral vertices.

5. Proof of Theorem B. Let $M$ be a compact symplectic manifold with a Hamiltonian action of a compact Lie group $G$. In this section, we fix a (possibly non-integral) symplectic class $\left[\omega_{0}\right]$, and consider the set of all $G$-invariant symplectic forms in the class $\left[\omega_{0}\right]$. Two $G$-invariant symplectic forms $\omega_{0}, \omega_{1}$ in the class are joined by a path

$$
\omega_{t}=\omega_{0}+t d \alpha, \quad 0 \leq t \leq 1
$$

where $\alpha$ is a $G$-invariant 1-form on $M$, and $\omega_{t}$ is a 2-form on $M$ which is not necessarily symplectic in general. Let $\mu_{X, 0}$ be a Hamiltonian function for $X \in \mathfrak{g}$ with respect to $\omega_{0}$ defined by $d \mu_{X, 0}=i(X) \omega_{0}$. Put

$$
\mu_{X, t}=\mu_{X, 0}-t \alpha(X), \quad 0 \leq t \leq 1 .
$$

Proposition 5.1. For each $X \in \mathfrak{g}$, the function $\mu_{X, t}$ is a Hamiltonian function for $X$ with respect to $\omega_{t}$, i.e., for all $0 \leq t \leq 1$,

$$
d \mu_{X, t}=i(X) \omega_{t}
$$

and it satisfies

$$
\int_{M} \mu_{X, t} \omega_{t}^{n}=\int_{M} \mu_{X, 0} \omega_{0}^{n}
$$


Moreover, for any point $p \in M$ in the zeroes of $X$, the value $\mu_{X, t}(p)$ depends only on $p$ and is independent of $t$.

Proof. For $p$ in the zeroes of $X$, the value $\mu_{X, t}(p)$ is independent of $t$ by (5.1). Furthermore, from $L_{X} \alpha=0$, we obtain (5.2) as follows:

$$
d \mu_{X, t}=d \mu_{X, 0}-t d\{\alpha(X)\}=i(X) \omega_{0}+t i(X) d \alpha=i(X) \omega_{t} .
$$

Hence, the proof is reduced to showing (5.3). It then suffices to show that the derivative with respect to $t$ of the left-hand side of (5.3) vanishes identically. In fact, by (5.2),

$$
\frac{d}{d t} \int_{M} \mu_{X, t}\left(\omega_{0}+t d \alpha\right)^{n}=\int_{M}\left\{-\alpha(X) \omega_{t}^{n}+\mu_{X, t} n d \alpha \wedge \omega_{t}^{n-1}\right\}
$$

where the right-hand side is $\int_{M}\left\{-\alpha(X) \omega_{t}^{n}-d \mu_{X, t} \wedge n \alpha \wedge \omega_{t}^{n-1}\right\}=\int_{M}\left\{-\alpha(X) \omega_{t}^{n}+\right.$ $\left.\alpha \wedge i(X) \omega_{t}^{n}\right\}=-\int_{M} i(X)\left\{\alpha \wedge \omega_{t}^{n}\right\}=0$, as required.

THEOREM 5.2. Let $\mu: M \rightarrow \mathfrak{g}^{*}$ be the reduced moment map for a symplectic form $\omega$ in the class $\left[\omega_{0}\right]$. For each real-valued smooth function $\phi=\phi\left(x_{1}, \cdots, x_{\ell}\right)$ on $\mathbb{R}^{\ell}$, the function $\Phi_{\ell}^{\phi}: \mathfrak{g}^{\ell} \rightarrow \mathbb{R}$ defined by $\Phi_{\ell}^{\phi}\left(Y_{1}, \cdots, Y_{\ell}\right):=\int_{M} \phi\left(\mu_{Y_{1}}, \ldots, \mu_{Y_{\ell}}\right) \omega^{n}$ is independent of the choice of $\omega$ in the class $\left[\omega_{0}\right]$.

Proof. Let $\omega_{t}=\omega_{0}+t d \alpha, 0 \leq t \leq 1$, be the path between $\omega_{0}$ and $\omega$, where $\omega_{1}=\omega$. For each $Y \in \mathfrak{g}$, the Hamiltonian function $\mu_{Y, t}$ for $Y$ with respect to $\omega_{t}$ with normaliztion

$$
\int_{M} \mu_{Y, t} \omega_{t}^{n}=0
$$

is written in the form (5.1) by Proposition 5.1. Put $\phi_{j}:=\partial \phi / \partial x_{j}$ and $\mu_{j, t}:=\mu_{Y_{j}, t}$ for simplicity. Then, for all $0 \leq t \leq 1$,

$$
\begin{aligned}
& \frac{d}{d t} \int_{M} \phi\left(\mu_{1, t}, \cdots, \mu_{\ell, t}\right) \omega_{t}^{n} \\
= & -\int_{M} \sum_{j=1}^{\ell} \phi_{j}\left(\mu_{1, t}, \cdots, \mu_{\ell, t}\right) \alpha\left(Y_{j}\right) \omega_{t}^{n}+\int_{M} \phi\left(\mu_{1, t}, \cdots, \mu_{\ell, t}\right) d \alpha \wedge n \omega_{t}^{n-1} \\
= & -\int_{M} \sum_{j=1}^{\ell} \phi_{j}\left(\mu_{1, t}, \cdots, \mu_{\ell, t}\right) \alpha\left(Y_{j}\right) \omega_{t}^{n}-\int_{M} d\left\{\phi\left(\mu_{1, t}, \cdots, \mu_{\ell, t}\right)\right\} \wedge \alpha \wedge n \omega_{t}^{n-1} \\
= & -\int_{M} \sum_{j=1}^{\ell} \phi_{j}\left(\mu_{1, t}, \cdots, \mu_{\ell, t}\right)\left\{\alpha\left(Y_{j}\right)-\alpha \wedge d \mu_{j, t} \wedge n \omega_{t}^{n-1}\right\} \\
= & -\int_{M} \sum_{j=1}^{\ell} \phi_{j}\left(\mu_{1, t}, \cdots, \mu_{\ell, t}\right) i\left(Y_{j}\right)\left(\alpha \wedge \omega_{t}^{n}\right)=0,
\end{aligned}
$$

i.e., $\int_{M} \phi\left(\mu_{Y_{1}}, \ldots, \mu_{Y_{\ell}}\right) \omega^{n}$ is independent of the choice of $\omega$ in the class $\left[\omega_{0}\right]$.

Proof of Theorem B. (a) immediately follows from Theorem 5.2 applied to $\phi=$ $\Pi_{j=1}^{\ell} x_{j}$. 
(b) Assume that $[\omega]$ is an integral class. For the $S^{1}$-action on $M$ generated by $0 \neq$ $X \in \mathfrak{g}_{\mathbb{Q}}$, we apply Proposition 3.1 to $C=0$. In view of Proposition $\mathrm{A}$, we have

$$
\delta_{\gamma}(X, 0, \ell) \in \mathbb{Q}, \quad \gamma \in \Gamma
$$

because by $0 \neq X \in \mathfrak{g}_{\mathbb{Q}}$, all eigenvalues of $L X_{i} / 2 \pi \sqrt{-1}$ are rational numbers. Then by Proposition 3.1, $\Phi_{\ell}(X, X, \cdots, X)=\int_{M}\left(\mu_{X}\right)^{\ell} \omega^{n} \in \mathbb{Q}$, as required.

Remark 5.3. (a) Let $0 \neq Y \in \mathfrak{g}$. Then the closure of $\{\exp (t Y) ; t \in \mathbb{R}\}$ in the compact Lie group $G$ is a compact torus whose dimension is denoted by $k(Y)$. Then it is easily seen that $k(Y)=1$ if and only if $Y \in \mathfrak{g}_{\mathbb{Q}}$.

(b) By the $G$-equivariance of the moment map $\mu$, the symmetric polynomial $\Phi_{\ell}$ in (a) of Theorem B is an invariant polynomial in $\mathfrak{g}^{*}$ for the coadjoint action of $G$ on $\mathfrak{g}^{*}$.

(c) In the situation of (b) of Theorem B, suppose that $G$ is the torus $T=\left(S^{1}\right)^{k}$. Then obviously, $\Phi_{\ell}$ is defined over $\mathbb{Q}$, i.e., $\Phi_{\ell}$ takes values in $\mathbb{Q}$ on $\otimes^{\ell} \mathfrak{t}_{\mathbb{Q}}$.

REMARK 5.4. Replacing the localization principle by the method of equivariant cohomologies, we have an alternative proof for both Proposition A and (b) of Theorem B. We also have another proof for (a) of Theorem B if $[\omega]$ is integral. To see these, we first assume the situation in Proposition A, so that $[\omega]$ is integral and the action of $T=\left(S^{1}\right)^{k}$ on $M$ lifts to a bundle action on $P$. As in [11], let $E T \rightarrow B T$ be the universal $T$-bundle. Put $M T:=E T \times_{T} M$. Then $P T:=E T \times_{T} P$ is the associated principal bundle over $M T$. Then via the identification of $I^{\ell}(T)$ with $H^{2 \ell}(B T ; \mathbb{C})$, we have the linear map $\operatorname{pr}_{*} \circ W: I^{n+\ell}\left(S^{1}\right) \rightarrow I^{\ell}(T)$ such that the diagram

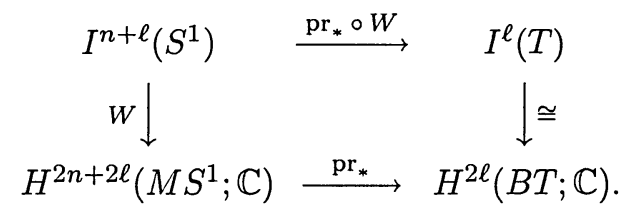

commutes, where $W$ is the Weil homomorphism associated to $P T \rightarrow M T$, and $\mathrm{pr}_{*}$ is the Gysin map for pr : $M T \rightarrow B T$. Let $\nu: M \rightarrow \mathfrak{g}^{*}$ be the moment map (as in (a) of Theorem 2.5) for $(M, \omega)$ such that $\nu(M)$ is an integral convex polytope. Then

$$
\int_{M}\left(\nu_{Y}\right)^{j} \omega^{n}=\frac{n ! j !}{(n+j) !}\left\{\left(\operatorname{pr}_{*} \circ W\right)\left(c_{1}^{n+j}\right)\right\}(Y, \cdots, Y), \quad Y \in \mathfrak{t}
$$

for each nonnegative integer $j$. Let $\mu: M \rightarrow \mathfrak{g}$ be the reduced moment map. Put $C:=\left\{\int_{M} \omega^{n}\right\}^{-1} \int_{M} \nu_{Y} \omega^{n}$. By $\mu_{Y}=\nu_{Y}-C$, we have

$$
\int_{M}\left(\mu_{Y}\right)^{\ell} \omega^{n}=\sum_{j=0}^{\ell} \frac{\ell !(-C)^{\ell-j}}{j !(\ell-j) !} \int_{M}\left(\nu_{Y}\right)^{j} \omega^{n} .
$$

Now, let $0 \neq Y \in \mathfrak{t}_{\mathbb{Z}}$, and we consider the subtorus $T^{1}:=S^{1}=\{\exp t Y ; t \in \mathbb{R}\}$ of $T$ generated by $Y$. Replacing $T$ by this one-dimensional torus $T^{1}$ in the above diagram with cohomologies considered over $\mathbb{Z}$, we obtain

$$
\begin{aligned}
H^{2 \ell}\left(B T^{1} ; \mathbb{Z}\right) & \cong \mathbb{Z} \\
\left(\operatorname{pr}_{*} \circ W\right)\left(c_{1}^{n+\ell}\right) & \leftrightarrow f_{c_{1}^{n+\ell}}^{L}(Y)
\end{aligned}
$$


where $\mathrm{pr}_{*}$ denotes the corresponding Gysin map of $H^{2 n+2 \ell}\left(M S^{1} ; \mathbb{Z}\right)$ to $H^{2 \ell}\left(B T^{1} ; \mathbb{Z}\right)$ by abuse of terminology. Hence, for $\ell=1$,

$$
\int_{M} \nu_{Y} \omega^{n}=-(n+1)^{-1} f_{c_{1}^{n+1}}^{L}(Y) \in \mathbb{Q}
$$

Since $[\omega]$ is integral, we obtain $C \in \mathbb{Q}$. Then by $\mu_{Y}=\nu_{Y}-C$ and (a) of Theorem 2.5 , we now see that $\mu\left(M^{T}\right) \subset \mathfrak{t}_{\mathbb{Q}}^{*}$, as required.

We next consider the situation in (b) of Theorem B. Let $0 \neq X \in \mathfrak{g}_{\mathbb{Q}}$. Then for some positive integer $m$, the multiple $Y:=m X$ satisfies $\exp Y=1$, so that $Y$ is in the lattice for the one-dimensional torus $T^{1}$ generated by $Y$. Then by the argument above,

$$
\frac{(n+\ell) !}{n ! \ell !} \int_{M}\left(\nu_{Y}\right)^{\ell} \omega^{n}=f_{c_{1}^{n+\ell}}^{L}(Y) \in \mathbb{Z}, \quad \ell=0,1,2, \ldots
$$

Hence, in view of $\mu_{Y}=m \mu_{X}$ together with $m \in \mathbb{Z}_{+}$and (5.5) above, we obtain $\int_{M}\left(\mu_{X}\right)^{\ell} \omega^{n} \in \mathbb{Q}$, as required.

By a similar method, we now prove (a) of Theorem B under the assumption that $[\omega]$ is integral. Let $Y_{1}, \ldots, Y_{\ell} \in \mathfrak{g}$, and let $s_{1}, \ldots, s_{\ell} \in \mathbb{R}$. Put $Y:=s_{1} Y_{1}+\cdots+s_{\ell} Y_{\ell}$. Then by (a) of Remark 5.3, $Y \in \mathfrak{t}$ for some toral subalgebra $\mathfrak{t}$ of $\mathfrak{g}$. Since the righthand side of (5.4) is independent of the choice of $\omega$ in $\mathcal{S}$, we now see from (5.4) and (5.5) that the left-hand side $\int_{M}\left(s_{1} \mu_{Y_{1}}+\cdots+s_{\ell} \mu_{Y_{\ell}}\right)^{\ell} \omega^{n}$ of (5.5) is independent of the choice of $\omega$ in the symplectic class. Since $s_{1}, \ldots, s_{\ell}$ are arbitrary, its coefficient $\int_{M} \mu_{Y_{1}} \cdots \mu_{Y_{\ell}} \omega^{n}$ in $s_{1} \cdots s_{\ell}$ is also independent of the choice of $\omega$ in the class, as required.

6. Complex manifolds with symplectic forms of type $(1,1)$. Finally, we consider a compact complex manifold $M$ of complex dimension $n$ endowed with a pseudo-Kähler form $\omega_{0}$, i.e., a possibly non-Kähler symplectic form $\omega_{0}$ of type $(1,1)$. For $\mathcal{S}$ as in the introduction, each element $\omega$ in $\mathcal{S}$ is written locally in the form

$$
\omega=\frac{\sqrt{-1}}{2 \pi} \sum_{\alpha, \beta} g_{\alpha \bar{\beta}} d z^{\alpha} \wedge d \bar{z}^{\beta}
$$

for a system $\left(z^{1}, z^{2}, \ldots, z^{n}\right)$ of holomorphic local coordinates on $M$. Here $\operatorname{det}\left(g_{\alpha \bar{\beta}}\right)$ is nonvanishing wherever it is defined. To each $u \in C^{\infty}(M)_{\mathbb{C}}$, we can associate a complex vector field $\operatorname{grad}_{\omega}^{\mathbb{C}} u$ of type $(1,0)$ on $M$ by

$$
\operatorname{grad}_{\omega}^{\mathbb{C}} u:=\frac{2 \pi}{\sqrt{-1}} \sum_{\alpha, \beta} g^{\bar{\beta} \alpha} \frac{\partial u}{\partial \bar{z}^{\beta}} \frac{\partial}{\partial z^{\alpha}} .
$$

Let $\mathfrak{h}_{\omega}$ denote the space of all holomorphic vector fields on $M$ expressible as $\operatorname{grad}_{\omega}^{\mathbb{C}} v$ for some $v \in C^{\infty}(M)_{\mathbb{C}}$, where we always normalize $v$ in such a way that $\int_{M} v \omega^{n}=0$. By the notation in the introduction, all $\omega$ in $\mathcal{S}$ are written as $\omega_{\varphi}$ for some $\varphi \in C^{\infty}(M)_{\mathbb{R}}$. Then we shall show that every element $\xi=\operatorname{grad}_{\omega_{0}}^{\widetilde{C}} v_{0}$ in $\mathfrak{h}$ is rewritten as

$$
\xi=\operatorname{grad}_{\omega}^{\mathbb{C}} v, \quad \text { where } v:=v_{0}+(\sqrt{-1} / 2 \pi) \xi \varphi .
$$

Proof. For $0 \leq t \leq 1$, put $\omega(t):=\omega_{0}+(\sqrt{-1} / 2 \pi) t \partial \bar{\partial} \varphi$ and $v(t):=v_{0}+$ $(\sqrt{-1} / 2 \pi) t \xi \varphi$. Then by the same computation as in $[10 ; \mathrm{p} .208]$, we obtain $i(\xi) \omega(t)=$ 
$\bar{\partial} v(t)$ for all $t$. Hence, the proof is reduced to showing $q(t):=\int_{M} v(t) \omega(t)^{n}$ vanishes for all $t$.

$$
\begin{aligned}
\dot{q}(t) & =\int_{M}(\sqrt{-1} / 2 \pi)\left\{(i(\xi) \partial \varphi) \omega(t)^{n}+v(t) \partial \bar{\partial} \varphi \wedge n \omega(t)^{n-1}\right\} \\
& =\int_{M}(\sqrt{-1} / 2 \pi)\left\{\partial \varphi \wedge(i(\xi) \omega(t)) \wedge n \omega(t)^{n-1}+\bar{\partial} v(t) \wedge \partial \varphi \wedge n \omega(t)^{n-1}\right\} \\
& =0
\end{aligned}
$$

where we used the identities $\xi \varphi=i(\xi) \partial \varphi$ and $i(\xi) \omega(t)=\bar{\partial} v(t)$. Since $q(0)=$ $\int_{M} v_{0} \omega_{0}^{n}=0$, we now obtain $q(t)=0$ for all $t$, as required.

Now by (6.1), we obtain $\mathfrak{h}_{\omega}=\mathfrak{h}_{\omega_{0}}$. Hence, $\mathfrak{h}_{\omega}$ is independent of the choice of $\omega$ in $\mathcal{S}$, and is simply written as $\mathfrak{h}$ in this section (see (6.2) below for another characterization as in the introduction). Moreover, let $G_{0}$ denote the identity component $\operatorname{Aut}^{0}(M)$ of the group of all holomorphic automorphisms of $M$. Then the associated Lie algebra is the space $\mathfrak{g}_{0}:=H^{0}\left(M, \mathcal{O}\left(T^{1,0} M\right)\right)$ of all holomorphic vector fields on $M$. Identify $H^{1}(M, \mathcal{O})$ with the Dolbeault cohomology group $H^{0,1}(M)$. Then for the natural complex Lie group homomorphism

$$
\rho: G_{0} \rightarrow \operatorname{GL}\left(H^{1}(M, \mathcal{O}), \mathbb{C}\right)
$$

and the associated Lie algebra homomorphism $\rho_{*}: \mathfrak{g}_{0} \rightarrow \mathfrak{g l}_{\mathbb{C}}\left(H^{1}(M, \mathcal{O})\right)$, we put $\mathfrak{g}_{1}:=\operatorname{Ker} \rho_{*}$ and $\mathfrak{g}_{2}:=\mathfrak{g}_{1} \cap \mathfrak{h}$. Then $\mathfrak{g}_{0} \supset \mathfrak{g}_{1} \supset \mathfrak{g}_{2}$ and $\mathfrak{g}_{0} / \mathfrak{g}_{1} \cong$ Image $\rho_{*}$. We further obtain

THEOREM 6.1. (a) Both $\mathfrak{g}_{1}$ and $\mathfrak{g}_{2}$ are ideals of the complex Lie algebra $\mathfrak{g}_{0}$.

(b) $\left[\mathfrak{g}_{1}, \mathfrak{g}_{1}\right] \subset \mathfrak{g}_{2}$. In paricular, $\mathfrak{g}_{1} / \mathfrak{g}_{2}$ is an abelian Lie algebra.

(c) In view of the natural inclusions $\mathfrak{g}_{1} / \mathfrak{g}_{2} \subset \mathfrak{g}_{0} / \mathfrak{h} \subset H^{1}(M, \mathcal{O})$, the quotient $\mathfrak{g}_{1} / \mathfrak{g}_{2}$ is regarded as a complex vector subspace of $H^{1}(M, \mathcal{O})$.

Before proving this theorem, we consider the following situation. Let us choose a fine open cover $\mathcal{U}=\left\{U_{\lambda}\right\}_{\lambda \in \Lambda}$ of $M=\cup_{\lambda \in \Lambda} U_{\lambda}$ in such a way that each $U_{\lambda}$ is a sufficiently small Stein open subset of $M$ with a system $z_{\lambda}=\left(z_{\lambda}^{1}, z_{\lambda}^{2}, \ldots, z_{\lambda}^{n}\right)$ of holomorphic local coordinates. Let $\xi \in \mathfrak{g}_{0}$ and on each $U_{\lambda}$, we write

$$
\xi=\frac{2 \pi}{\sqrt{-1}} \sum_{\alpha} a_{\alpha}^{\lambda} \frac{\partial}{\partial z_{\lambda}^{\alpha}}
$$

By $\bar{\partial}(i(\xi) \omega)=-i(\xi) \bar{\partial} \omega=0$, we can write $i(\xi) \omega=\bar{\partial} u_{\lambda}$ for some $u_{\lambda} \in C^{\infty}\left(U_{\lambda}\right)_{\mathbb{C}}$, $\lambda \in \Lambda$. Then on $U_{\lambda}$, using the metric tensor of the pseudo-Kähler form $\omega$, we have

$$
a_{\alpha}^{\lambda}=\sum_{\beta} g_{\lambda}^{\alpha \bar{\beta}} \frac{\partial u_{\lambda}}{\partial \bar{z}_{\lambda}^{\beta}}, \quad \alpha=1,2, \ldots, n .
$$

Since $\bar{\partial} u_{\nu}-\bar{\partial} u_{\lambda}=i(\xi) \omega-i(\xi) \omega=0$ on $U_{\lambda} \cap U_{\nu}$, by setting $u_{\lambda \nu}:=u_{\nu}-u_{\lambda} \in H^{0}\left(U_{\lambda} \cap\right.$ $\left.U_{\nu}, \mathcal{O}\right)$, we have a Cĕch 1 -cocycle $\left(u_{\lambda \nu}\right) \in Z^{1}(\mathcal{U}, \mathcal{O})$ and the corresponding class $\left[\left(u_{\lambda \nu}\right)\right] \in H^{1}(M, \mathcal{O})$. We now have a natural map $\phi: \mathfrak{g}_{0} \rightarrow H^{1}(M, \mathcal{O})$ which sends $\xi \in \mathfrak{g}_{0}$ to $\phi(\xi):=\left[\left(u_{\lambda \nu}\right)\right] \in H^{1}(M, \mathcal{O})$. Now, in terms of the Dolbeault cohomology, this $\phi(\xi)$ is written as the class $[i(\xi) \omega] \in H^{1}(M, \mathcal{O})$ in the introduction. We now claim that

$\operatorname{Ker} \phi=\mathfrak{h}$. 
Since the inclusion $\mathfrak{h} \subset \operatorname{Ker} \phi$ obviously holds, it suffices to show $\operatorname{Ker} \phi \subset \mathfrak{h}$. Let $\xi \in \operatorname{Ker} \phi$. Then the Dolbeault cohomology class $[i(\xi) \omega]$ vanishes, i.e., $i(\xi) \omega$ is written as $\bar{\partial} \tilde{u}$ on $M$ for some $\tilde{u} \in C^{\infty}(M)_{\mathbb{C}}$. Hence $\xi=\operatorname{grad}_{\omega}^{\mathbb{C}} \tilde{u}$ and Ker $\phi \subset \mathfrak{h}$, as required.

Proof of (c). By (6.2) above, we immediate obtain $\mathfrak{g}_{1} / \mathfrak{g}_{2}=\mathfrak{g}_{1} /\left(\mathfrak{g}_{1} \cap \mathfrak{h}\right)=\left(\mathfrak{g}_{1}+\right.$ $\mathfrak{h}) / \mathfrak{h} \subset \mathfrak{g}_{0} / \mathfrak{h}=$ Image $\phi \subset H^{1}(M, \mathcal{O})$, as required.

Proof of (a). Since $\mathfrak{g}_{1}=\operatorname{Ker} \rho_{*}$, the Lie algebra $\mathfrak{g}_{1}$ is obviously an ideal of the Lie algebra $\mathfrak{g}_{0}$. Next, let $\xi \in \mathfrak{g}_{0}$ and $\zeta \in \mathfrak{g}_{2}$. Then $i(\xi) \omega=\bar{\partial} u_{\lambda}$ for some $u_{\lambda} \in C^{\infty}\left(U_{\lambda}\right)_{\mathbb{C}}$ on each $U_{\lambda}$. We further have $\zeta=\operatorname{grad}_{\omega}^{\mathbb{C}} v$ for some $v \in C^{\infty}(M)_{\mathbb{C}}$. In terms of the symplectic form $\omega$, we consider the Poisson bracket

$$
\left[u_{\lambda}, v\right]:=2 \pi \sqrt{-1} \sum_{\alpha, \beta} g_{\lambda}^{\alpha \bar{\beta}}\left(\frac{\partial u_{\lambda}}{\partial z^{\alpha}} \frac{\partial v}{\partial \bar{z}^{\beta}}-\frac{\partial u_{\lambda}}{\partial \bar{z}^{\beta}} \frac{\partial v}{\partial z^{\alpha}}\right)=\xi v-\zeta u_{\lambda}
$$

on $U_{\lambda}, \lambda \in \Lambda$. Then $i([\xi, \zeta]) \omega=\bar{\partial}\left[u_{\lambda}, v\right]$ on each $U_{\lambda}$. Now by $\zeta \in \mathfrak{g}_{2} \subset \mathfrak{g}_{1}$, the 1-cocycle $\left(\zeta u_{\lambda \nu}\right) \in Z^{1}(\mathcal{U}, \mathcal{O})$ is cohomologous to zero, i.e., $\zeta u_{\lambda \nu}=w_{\nu}-w_{\lambda}$ on $U_{\lambda} \cap U_{\nu}$ for some $w_{\lambda} \in H^{0}\left(U_{\lambda}, \mathcal{O}\right), \lambda \in \Lambda$. Then by setting $\eta_{\lambda}:=\xi v-\zeta u_{\lambda}+w_{\lambda}, \lambda \in \Lambda$, we have a smooth function $\eta \in C^{\infty}(M)_{\mathbb{C}}$ such that

$$
\eta_{\mid U_{\lambda}}=\eta_{\lambda}, \quad \lambda \in \Lambda
$$

Then $i([\xi, \zeta]) \omega=\bar{\partial}\left[u_{\lambda}, v\right]=\bar{\partial}\left(\left[u_{\lambda}, v\right]+w_{\lambda}\right)=\bar{\partial} \eta_{\lambda}=\bar{\partial} \eta$. Hence, $[\xi, \zeta]=\operatorname{grad}_{\omega}^{\mathbb{C}} \eta$ and $[\xi, \zeta] \in \mathfrak{h}$. Since $\mathfrak{g}_{1}$ is an ideal of $\mathfrak{g}_{0}$, we therefore obtain $[\xi, \zeta] \in \mathfrak{g}_{1} \cap \mathfrak{h}=\mathfrak{g}_{2}$. This shows that $\mathfrak{g}_{2}$ is an ideal of $\mathfrak{g}_{0}$.

Proof of (b). Let $\xi, \xi^{\prime} \in \mathfrak{g}_{1}$. Then $i(\xi) \omega=\bar{\partial} u_{\lambda}$ and $i\left(\xi^{\prime}\right) \omega=\bar{\partial} u_{\lambda}^{\prime}$ for some $u_{\lambda}, u_{\lambda}^{\prime} \in C^{\infty}(M)_{\mathbb{C}}$ on each $U_{\lambda}$. In terms of the symplectic form $\omega$, we consider the Poisson bracket

$$
\left[u_{\lambda}, u_{\lambda}^{\prime}\right]=2 \pi \sqrt{-1} \sum_{\alpha, \beta} g_{\lambda}^{\alpha \bar{\beta}}\left(\frac{\partial u_{\lambda}}{\partial z^{\alpha}} \frac{\partial u_{\lambda}^{\prime}}{\partial \bar{z}^{\beta}}-\frac{\partial u_{\lambda}}{\partial \bar{z}^{\beta}} \frac{\partial u_{\lambda}^{\prime}}{\partial z^{\alpha}}\right)=\xi u_{\lambda}^{\prime}-\xi^{\prime} u_{\lambda}
$$

on $U_{\lambda}, \lambda \in \Lambda$. Then $i\left(\left[\xi, \xi^{\prime}\right]\right) \omega=\bar{\partial}\left[u_{\lambda}, u_{\lambda}^{\prime}\right]$ on each $U_{\lambda}$. By $\xi, \xi^{\prime} \in \mathfrak{g}_{1}$, the 1-cocycles $\left(\xi^{\prime} u_{\lambda \nu}\right),\left(\xi u_{\lambda \nu}^{\prime}\right) \in Z^{1}(\mathcal{U}, \mathcal{O})$ are cohomologous to zero (where $u_{\lambda \nu}:=u_{\nu^{\prime}}-u_{\lambda}, u_{\lambda \nu}^{\prime}:=$ $u_{\nu}^{\prime}-u_{\lambda}^{\prime}$ on $\left.U_{\lambda} \cap U_{\nu}\right)$, i.e., $\xi^{\prime} u_{\lambda \nu}=w_{\nu}-w_{\lambda}$ and $\xi u_{\lambda \nu}^{\prime}=w_{\nu}^{\prime}-w_{\lambda}^{\prime}$ on $U_{\lambda} \cap U_{\nu}$ for some $w_{\lambda}, w_{\lambda}^{\prime} \in H^{0}\left(U_{\lambda}, \mathcal{O}\right), \lambda \in \Lambda$. Then by setting $\tau_{\lambda}:=\xi u_{\lambda}^{\prime}-\xi^{\prime} u_{\lambda}-w_{\lambda}^{\prime}+w_{\lambda}, \lambda \in \Lambda$, we have a smooth function $\tau \in C^{\infty}(M)_{\mathbb{C}}$ such that

$$
\tau_{\mid U_{\lambda}}=\tau_{\lambda}, \quad \lambda \in \Lambda \text {. }
$$

Then $i\left(\left[\xi, \xi^{\prime}\right]\right) \omega=\bar{\partial}\left[u_{\lambda}, u_{\lambda}^{\prime}\right]=\bar{\partial}\left(\left[u_{\lambda}, u_{\lambda}^{\prime}\right]-w_{\lambda}^{\prime}+w_{\lambda}\right)=\bar{\partial} \tau_{\lambda}=\bar{\partial} \tau$. Hence $\left[\xi, \xi^{\prime}\right]=$ $\operatorname{grad}_{\omega}^{\mathbb{C}} \tau$ and $\left[\xi, \xi^{\prime}\right] \in \mathfrak{h}$. Since $\mathfrak{g}_{1}$ is an ideal of $\mathfrak{g}_{0}$, it now follows that $\left[\xi, \xi^{\prime}\right] \in \mathfrak{g}_{1} \cap \mathfrak{h}=\mathfrak{g}_{2}$, as required.

REMARK 6.2. In the above theorem, consider the special case where $(M, \omega)$ is a Kähler manifold. Then by the Hodge theory, $\rho$ above is a trivial map, and hence $\mathfrak{g}_{0}=\mathfrak{g}_{1}$. In particular, $\mathfrak{g}_{2}$ is the Lie subalgebra $\mathfrak{h}$ of $\mathfrak{g}_{0}$ associated to the kernel of the Jacobi homomorphism $\alpha: \operatorname{Aut}^{0}(M) \rightarrow \operatorname{Aut}^{0}(\operatorname{Alb}(M))(\cong \operatorname{Alb}(M))$ (cf. [6]).

Let $\xi_{1}, \xi_{2}, \ldots, \xi_{\ell} \in \mathfrak{h}$. By writing $\xi_{i}=\operatorname{grad}_{\omega}^{\mathbb{C}} v_{i}, i=1,2, \ldots, \ell$, as in (6.1), we define a multilinear form $\Phi_{\ell}: \otimes^{\ell} \mathfrak{h} \rightarrow \mathbb{C}$ by

$$
\Phi_{\ell}\left(\xi_{1}, \xi_{2}, \cdots, \xi_{\ell}\right)=\int_{M} v_{1} v_{2} \cdots v_{\ell} \omega^{n}
$$


TheOREM 6.3. The multilinear form $\Phi_{\ell}$ in (6.3) above can depend only on $\mathcal{S}$, and is independent of the choice of $\omega$ in $\mathcal{S}$.

Proof. For each $\omega \in \mathcal{S}$, we write $\omega=\omega_{\varphi}$ for some $\varphi \in C^{\infty}(M)_{\mathbb{R}}$. For $v_{i}$ above, put

$$
\begin{aligned}
& \omega(t):=\omega+(\sqrt{-1} / 2 \pi) t \partial \bar{\partial}(-\varphi) \\
& v_{i}(t):=v_{i}+(\sqrt{-1} / 2 \pi) t \xi_{i}(-\varphi), \quad i=1,2, \ldots, \ell
\end{aligned}
$$

for all $0 \leq t \leq 1$. As in the proof of (6.1), we have $\xi_{i}=\operatorname{grad}_{\omega(t)}^{\mathbb{C}} v_{i}(t)$. For each $i$, we put $\hat{v}_{i}(t):=\Pi_{j \neq i} v_{j}(t)$, where the product in the right-hand side is taken over all $j \in\{1,2, \ldots, \ell\}$ such that $j \neq i$. Put $\hat{v}(t):=\Pi_{i=1}^{\ell} v_{i}(t)$ and $q(t):=\int_{M} \hat{v}(t) \omega(t)^{n}$. Then

$$
\begin{aligned}
\dot{q}(t) & =-\int_{M}(\sqrt{-1} / 2 \pi)\left\{\hat{v}(t) \partial \bar{\partial} \varphi \wedge n \omega(t)^{n-1}+\sum_{i=1}^{\ell}\left(\xi_{i} \varphi\right) \hat{v}_{i}(t) \omega(t)^{n}\right\} \\
& =-\int_{M}(\sqrt{-1} / 2 \pi)\left\{\bar{\partial} \hat{v}(t) \wedge \partial \varphi \wedge n \omega(t)^{n-1}+\sum_{i=1}^{\ell}\left(i\left(\xi_{i}\right) \partial \varphi\right) \wedge \hat{v}_{i}(t) \omega(t)^{n}\right\} \\
& =-\int_{M}(\sqrt{-1} / 2 \pi)\left\{-\partial \varphi \wedge \bar{\partial} \hat{v}(t) \wedge n \omega(t)^{n-1}+\sum_{i=1}^{\ell}\left(i\left(\xi_{i}\right) \partial \varphi\right) \wedge \hat{v}_{i}(t) \omega(t)^{n}\right\} \\
& =-\int_{M}(\sqrt{-1} / 2 \pi) \sum_{i=1}^{\ell} i\left(\xi_{i}\right)\left\{\partial \varphi \wedge \hat{v}_{i}(t) \omega(t)^{n}+\partial \varphi \wedge \hat{v}_{i}(t) \omega(t)^{n}\right\}=0,
\end{aligned}
$$

where we used $i\left(\xi_{i}\right) \omega(t)=\bar{\partial} v_{i}(t)$ to obtain the last line. Since $\omega(0)=\omega_{0}$, we now conclude that the multilinear form $\Phi_{\ell}$ above is independent of the choice of $\omega$ in $\mathcal{S}$.

REMARK 6.4. More generally, given a complex-valued smooth function $\phi=$ $\phi\left(z_{1}, \cdots, z_{\ell}\right)$ on $\mathbb{C}^{\ell}$, the function $\Phi_{\ell}^{\phi}: \mathfrak{g}^{\ell} \rightarrow \mathbb{C}$ defined by $\Phi_{\ell}^{\phi}\left(Y_{1}, \cdots, Y_{\ell}\right)=$ $\int_{M} \phi\left(\mu_{Y_{1}}, \ldots \mu_{Y_{\ell}}\right) \omega^{n}$ is independent of the choice of $\omega$ in $\mathcal{S}$

REMARK 6.5. (a) Recall that the group $H$ in the introduction is the Lie subgroup of $\operatorname{Aut}^{0}(M)$ associated to the Lie subalgebra $\mathfrak{h}$ of $H^{0}\left(M, \mathcal{O}\left(T^{1,0}(M)\right)\right.$. Then $\Phi_{\ell}$ in (6.3) is regarded as an invariant polynomial in $\mathfrak{h}^{*}$ for the natural coadjoint action of $H$ on $\mathfrak{h}^{*}$.

(b) If $G$ is a compact real Lie subgroup of $H$, then the associated Lie algebra $\mathfrak{g}$ is regarded as a Lie subalgebra of $\mathfrak{h}$ by

$$
\mathfrak{g} \hookrightarrow \mathfrak{h}, \quad \xi_{\mathbb{R}} \mapsto \xi
$$

where each element in $\mathfrak{g}$ is written as $\xi_{\mathbb{R}}:=\xi+\bar{\xi}$ for some unique $\xi \in \mathfrak{h}$. We obviously see that the multilinear form $\Phi_{\ell}$ in (6.3) above, when restricted to $\otimes^{\ell} \mathfrak{g}$, coincides with the one in (a) of Theorem B.

For each $\omega \in \mathcal{S}$, let $\sigma(\omega)$ denote the scalar curvature of $\omega$ defined in Appendix. Moreover, to $\mathcal{S}$, we associate constants $c$ and $c_{0}$ as in Appendix. Recall that we fixed an element $\omega_{0}$ in $\mathcal{S}$. Then the functional $\mathcal{S} \ni \omega \mapsto c_{0} \kappa\left(\omega_{0}, \omega\right) \in \mathbb{R}$ is an analogue of the K-energy map (see (A.4) in Appendix). Obviously, $\omega \in \mathcal{S}$ is a critical point of 
this functional if and only if the scalar curvature $\sigma(\omega)$ is constant on $M$. As in [19; (5.5)] and $[10 ;(2.1)]$, the linear map $\mathcal{F}_{\mathcal{S}}: \mathfrak{h} \rightarrow \mathbb{C}$ defined by

$$
\mathcal{F}_{\mathcal{S}}(\xi):=\int_{M} v(\xi, \omega) \sigma(\omega) \omega^{n}=\int_{M} v(\xi, \omega)\{\sigma(\omega)-n c\} \omega^{n}, \quad \xi \in \mathfrak{h},
$$

is a Lie algebra character independent of the choice of $\omega$ in $\mathcal{S}$, where $v(\xi, \omega) \in C^{\infty}(M)_{\mathbb{C}}$ is such that the equalities $\xi=\operatorname{grad}_{\omega}^{\mathbb{C}} v(\xi, \omega)$ and $\int_{M} v(\xi, \omega) \omega^{n}=0$ are satisfied.

For the complex Lie group $\mathbb{C}^{*}=\{t ; 0 \neq t \in \mathbb{C}\}$, the associated Lie algebra is $\mathbb{C} \zeta \cong \mathbb{C}$, where $\zeta:=2 \pi \sqrt{-1} t \partial / \partial t$. Let $S^{1} \subset \mathbb{C}^{*}$ be the maximal compact torus. Then

LEMMA 6.6. Let $\iota: \mathbb{C}^{*} \hookrightarrow H$ be a complex Lie subgroup such that some symplectic form $\omega$ in $\mathcal{S}$ is preserved by the action of $\iota\left(S^{1}\right)$. If the symplectic class of $\mathcal{S}$ is integral, then the associated character $\iota_{*}: \mathbb{C} \rightarrow \mathfrak{g}$ satisfies $\mathcal{F}_{\mathcal{S}}\left(\iota_{*} \zeta\right) \in \mathbb{Q}$.

Proof. For the integral symplectic class of $\mathcal{S}$, we have a holomorphic line bundle $L$ on $M$ such that $\omega$ represents $c_{1}(L)$. Then by Proposition 2.1 , the action of $\iota\left(S^{1}\right)$ on $M$ lifts to a holomorphic bundle action on $L$. Let $n_{0}$ be a sufficiently large positive integer. For the anticanonical bundle $K_{M}^{-1}$ of $M$, put $L_{j}:=K_{M}^{-1} \otimes L^{n_{0}+n-2 j}$ for integers $j$. Then by $n_{0} \gg 1$, the classes $c_{1}\left(L_{j}\right)$ ?. $j=0,1, \ldots, n$, admit symplectic forms which are preserved by the action of $\iota\left(S^{1}\right)$. Now, the real vector field $X:=\zeta+\bar{\zeta}$, when restricted to $S^{1} \subset \mathbb{C}^{*}$, is a generator of the lattice $\mathbb{Z}$ in the Lie algebra $(\cong \mathbb{R})$ of $S^{1}$. Hence,

$$
\mathcal{F}_{\mathcal{S}}\left(\iota_{*} \zeta\right)=\frac{n c}{n+1} f_{c_{1}^{n+1}}^{L}\left(\iota_{*} X\right)-\sum_{j=0}^{n} \frac{(-1)^{j}}{2^{n}(n+1) !}\left(\begin{array}{c}
n \\
j
\end{array}\right) f_{c_{1}^{n+1}}^{L_{j}}\left(\iota_{*} X\right) \in \mathbb{Q},
$$

where we obtained the last equality by translating, word for word, Nakagawa's modified version $[22 ;(3.2)]$ of Tian's formula to our case.

Proof of Theorem $C$. Let $\mathfrak{z}(\mathfrak{k})$ be the center of $\mathfrak{k}$. Since the identity component of the center of $K$ fixes some element $\omega$ of $\mathcal{S}$, every element $\eta$ in $\mathfrak{z}(\mathfrak{k})$ is written as $\operatorname{grad}_{\omega}^{\mathbb{C}} u_{\eta}$ for some real-valued smooth function $u_{\eta}$ on $M$. Then the restriction of the bilinear form $\Phi_{2}: \otimes^{2} \mathfrak{h} \rightarrow \mathbb{C}$ to $\otimes^{2} \mathfrak{z}(\mathfrak{k})$ defines a positive definite real-valued quadratic form on $\mathfrak{z}(\mathfrak{k})$. Hence, there exists a unique element $\xi_{\mathcal{S}}$ of $\mathfrak{z}(\mathfrak{k})$ such that $\mathcal{F}_{\mathcal{S}}(\zeta)=\Phi_{2}\left(\zeta, \xi_{\mathcal{S}}\right)$ for all $\zeta \in \mathfrak{z}(\mathfrak{k})$. Moreover, as in $[9 ;(2.1)], \Phi_{2}\left(\left[\mathfrak{k}_{\mathbb{C}}, \mathfrak{k}_{\mathbb{C}}\right], \mathfrak{z}(\mathfrak{k})\right)=\Phi_{2}\left(\mathfrak{k}_{\mathbb{C}},\left[\mathfrak{k}_{\mathbb{C}}, \mathfrak{z}(\mathfrak{k})\right]\right)=\{0\}=$ $\mathcal{F}_{\mathcal{S}}\left(\left[\mathfrak{k}_{\mathbb{C}}, \mathfrak{k}_{\mathbb{C}}\right]\right)$. Hence, we have

$$
\mathcal{F}_{\mathcal{S}}(\zeta)=\Phi_{2}\left(\zeta, \xi_{\mathcal{S}}\right) \quad \text { for all } \zeta \in \mathfrak{k}_{\mathbb{C}}
$$

Now the same arguments as in $[9 ;(3.3)]$ show that, if the symplectic class of $\mathcal{S}$ is integral, then by Lemma 6.6, Remark 5.3 (c) and Remark 6.5 (b), we have $\exp \left(\nu \xi_{\mathcal{S}}\right)=$ 1 for some positive integer $\nu$, as required.

Proof of Corollary D. (a) Let $\omega$ be strictly extremal. Then $\operatorname{Ad}(h) \tau_{\omega} \in \mathfrak{k}$ for some $h \in H$. Further by $(6.2), \mathcal{F}_{\mathcal{S}}(\zeta)=\Phi_{2}\left(\zeta, \tau_{\omega}\right)$ for all $\zeta \in \mathfrak{h}$. Hence, if $\zeta \in \mathfrak{k}_{\mathbb{C}}$, then by setting $\zeta^{\prime}:=\operatorname{Ad}\left(h^{-1}\right) \zeta$, we have

$$
\Phi_{2}\left(\zeta, \xi_{\mathcal{S}}\right)=\mathcal{F}_{\mathcal{S}}(\zeta)=\mathcal{F}_{\mathcal{S}}\left(\zeta^{\prime}\right)=\Phi_{2}\left(\zeta^{\prime}, \tau_{\omega}\right)=\Phi_{2}\left(\operatorname{Ad}(h) \zeta^{\prime}, \operatorname{Ad}(h) \tau_{\omega}\right)=\Phi_{2}\left(\zeta, \operatorname{Ad}(h) \tau_{\omega}\right) .
$$

Thus, $\xi_{\mathcal{S}}=\operatorname{Ad}(h) \tau_{\omega}$. Now by Theorem $\mathrm{C}, \exp \left(\nu \tau_{\omega}\right)=1$ for some positive integer $\nu$. Then by Proposition A applied to the $S^{1}$ generated by $\tau_{\omega}$, both $\max _{M}\{\sigma(\omega)-n c\}$ and $\min _{M}\{\sigma(\omega)-n c\}$ are rational. Since $n c$ is rational, so are $\max _{M} \sigma(\omega)$ and $\min _{M} \sigma(\omega)$. 
(b) If $\omega$ is a Kähler form, then the one-parameter group $\left\{\exp \left(t \tau_{\omega}\right) ; t \in \mathbb{R}\right\}$ obviously sits in the compact group of the isometries of $(M, \omega)$. So, we next assume that $H$ is a reductive algebraic group, and it suffices to show $\tau_{\omega} \in \mathfrak{z}(\mathfrak{k})$. Then $\mathfrak{h}=\left[\mathfrak{k}_{\mathbb{C}}, \mathfrak{k}_{\mathbb{C}}\right] \oplus \mathfrak{z} \mathbb{C}(\mathfrak{k})$ for the complexification $\mathfrak{z} \mathbb{C}(\mathfrak{h})$ of $\mathfrak{z}(\mathfrak{k})$ in $\mathfrak{h}$. Further by

$$
\Phi_{2}\left(\left[\mathfrak{k}_{\mathbb{C}}, \mathfrak{k}_{\mathbb{C}}\right], \tau_{\omega}\right)=\mathcal{F}_{\mathcal{S}}\left(\left[\mathfrak{k}_{\mathbb{C}}, \mathfrak{k}_{\mathbb{C}}\right]\right)=\{0\},
$$

together with $\Phi_{2}\left(\left[\mathfrak{k}_{\mathbb{C}}, \mathfrak{k}_{\mathbb{C}}\right], \mathfrak{z} \mathbb{C}(\mathfrak{k})\right)=\Phi_{2}\left(\mathfrak{k}_{\mathbb{C}},\left[\mathfrak{k}_{\mathbb{C}}, \mathfrak{z} \mathbb{C}(\mathfrak{k})\right]\right)=\{0\}$, it follows that $\tau_{\omega} \in$ $\mathfrak{z} \mathbb{C}(\mathfrak{k})$. Since $\sigma(\omega)$ is a real-valued function, we obtain $\tau_{\omega} \in \mathfrak{z}(\mathfrak{k})$, as required.

REMARK 6.7. Let $M_{1}$ be a Fano manifold as in [16] with an extremal Kähler metric in the class $c_{1}\left(M_{1}\right)_{\mathbb{R}}$ such that $\operatorname{Aut}^{0}\left(M_{1}\right)$ is a reductive algebraic group. Put $M:=M_{1} \times M_{2}$ for a Kähler-Einstein manifold $M_{2}$ with $c_{1}(M)<0$, while $c_{1}(M)_{\mathbb{R}}$ is chosen as the symplectic class of $\mathcal{S}$. Then this gives a rather trivial example of Theorem $\mathrm{C}$ and Corollary D above.

Appendix. K-energy maps. In this appendix, we generalize K-energy maps to complex symplectic cases. Fix a compact complex connected manifold $M$ of complex dimension $n$ with a complex symplectic form $\omega_{0}$ of type $(1,1)$, i.e., with a $d$-closed $C^{\infty}$ $(1,1)$-form $\omega_{0}$ with complex coefficients such that $\omega_{0}^{n}$ is nowhere vanishing on $M$. Put $\omega_{\varphi}:=\omega_{0}+(\sqrt{-1} / 2 \pi) \partial \bar{\partial} \varphi$ for each complex-valued smooth function $\varphi \in C^{\infty}(M)_{\mathbb{C}}$. Let $\mathcal{S}_{\mathbb{C}}$ denote the set of all complex symplectic forms on $M$ expressible as $\omega_{\varphi}$ such that

$$
\left|\omega_{\varphi}^{n} / \omega_{0}^{n}\right|^{-1}\left(\omega_{\varphi}^{n} / \omega_{0}^{n}\right) \in \operatorname{Map}\left(M, S^{1}\right)
$$

is homotopic to a trivial map. Hence, if $\omega \in \mathcal{S}_{\mathbb{C}}$, then $\log \left(\omega^{n} / \omega_{0}^{n}\right)$ is a possibly complex-valued smooth function on $M$ which is uniquely determined by $\omega$ up to $2 \pi \sqrt{-1} \mathbb{Z}$. For each $\omega \in \mathcal{S}_{\mathbb{C}}$, we put $\operatorname{Ric}(\omega):=(\sqrt{-1} / 2 \pi) \bar{\partial} \partial \log \omega^{n}$, and let $\sigma(\omega)$ be the scalar curvature of $\omega$ defined by $\sigma(\omega):=n \operatorname{Ric}(\omega) \wedge \omega^{n-1} / \omega^{n}$. Put $c_{0}:=\int_{M} \omega^{n}$. Let $c$ be the constant

$$
c:=c_{0}^{-1} \int_{M} \operatorname{Ric}(\omega) \wedge \omega^{n-1} \in \mathbb{C},
$$

which is independent of the choice of $\omega$ in $\mathcal{S}_{\mathbb{C}}$. For $\omega, \omega^{\prime} \in \mathcal{S}_{\mathbb{C}}$, there exists $\zeta \in$ $C^{\infty}(M)_{\mathbb{C}}$ such that $\omega^{\prime}=\omega+(\sqrt{-1} / 2 \pi) \partial \bar{\partial} \zeta$. As in the explicit formula of Bando [2] (see also [12]) for the K-energy map in Kähler cases, we can similarly define a functional $\kappa: \mathcal{S}_{\mathbb{C}} \times \mathcal{S}_{\mathbb{C}} \rightarrow \mathbb{C} / \sqrt{-1} \mathbb{Z}$ by setting, modulo $\sqrt{-1} \mathbb{Z}$,

$$
\begin{aligned}
& \kappa\left(\omega, \omega^{\prime}\right) \\
:= & c_{0}^{-1} \int_{M}\left\{\frac{\log \left(\omega^{\prime n} / \omega^{n}\right)}{2 \pi} \omega^{\prime n}-\zeta \operatorname{Ric}(\omega) \sum_{i=0}^{n-1} \omega^{n-1-i}{\omega^{\prime}}^{i}+\frac{n c \zeta}{n+1} \sum_{i=0}^{n} \omega^{n-i} \omega^{\prime i}\right\} .
\end{aligned}
$$

LEMma A.1. The functional $\kappa$ satisfies the 1-cocycle conditions, i.e., for all $\omega$, $\omega^{\prime}, \omega^{\prime \prime} \in \mathcal{S}_{\mathbb{C}}$, the following equalities hold:

(a) $\kappa(\omega, \omega)=0$;

(b) $\kappa\left(\omega, \omega^{\prime}\right)+\kappa\left(\omega^{\prime}, \omega^{\prime \prime}\right)=\kappa\left(\omega, \omega^{\prime \prime}\right)$.

Proof. (a) is straightforward from the definition of $c$. To see (b), let $\tilde{\mathcal{S}}_{\mathbb{C}}$ denote the set of all $\varphi \in C^{\infty}(M)_{\mathbb{C}}$ such that $\omega_{\varphi} \in \mathcal{S}_{\mathbb{C}}$. Then for $\varphi, \varphi^{\prime} \in \tilde{\mathcal{S}}_{\mathbb{C}}$ with $\omega=\omega_{\varphi}$ and $\omega^{\prime}=\omega_{\varphi^{\prime}}$

$$
\kappa\left(\omega, \omega^{\prime}\right)=c_{0}^{-1} \kappa_{1}\left(\varphi, \varphi^{\prime}\right)+(n+1)^{-1} c_{0}^{-1} n c \kappa_{2}\left(\varphi, \varphi^{\prime}\right)
$$


modulo $\sqrt{-1} \mathbb{Z}$, where $\kappa_{1}: \tilde{\mathcal{S}}_{\mathbb{C}} \times \tilde{\mathcal{S}}_{\mathbb{C}} \rightarrow \mathbb{C} / \sqrt{-1} c_{0} \mathbb{Z}$ and $\kappa_{2}: \tilde{\mathcal{S}}_{\mathbb{C}} \times \tilde{\mathcal{S}}_{\mathbb{C}} \rightarrow \mathbb{C}$ are the functionals defined by setting

$$
\begin{aligned}
& \kappa_{1}\left(\varphi, \varphi^{\prime}\right):=\int_{M}\left\{\frac{\log \left(\omega^{\prime n} / \omega^{n}\right)}{2 \pi} \omega^{\prime n}-\left(\varphi^{\prime}-\varphi\right) \operatorname{Ric}(\omega) \sum_{i=0}^{n-1} \omega^{n-1-i} \omega^{\prime i}\right\} \\
& \kappa_{2}\left(\varphi, \varphi^{\prime}\right):=\int_{M}\left(\varphi^{\prime}-\varphi\right) \sum_{i=0}^{n} \omega^{n-i} \omega^{\prime i}
\end{aligned}
$$

for all $\varphi, \varphi^{\prime} \in \tilde{\mathcal{S}}_{\mathbb{C}}$. Here, in the first identity for $\kappa_{1}$, the right-hand side is taken modulo $\sqrt{-1} c_{0} \mathbb{Z}$. Now, the proof of (b) above is reduced to showing the 1-cocycle conditions

$$
\begin{aligned}
& \kappa_{1}\left(\varphi, \varphi^{\prime}\right)+\kappa_{1}\left(\varphi^{\prime}, \varphi^{\prime \prime}\right)=\kappa_{1}\left(\varphi, \varphi^{\prime \prime}\right) \\
& \kappa_{2}\left(\varphi, \varphi^{\prime}\right)+\kappa_{2}\left(\varphi^{\prime}, \varphi^{\prime \prime}\right)=\kappa_{2}\left(\varphi, \varphi^{\prime \prime}\right)
\end{aligned}
$$

for all $\varphi, \varphi^{\prime}, \varphi^{\prime \prime} \in \tilde{\mathcal{S}}_{\mathbb{C}}$. For simplicity, put $\zeta=\varphi^{\prime}-\varphi, \zeta^{\prime}=\varphi^{\prime \prime}-\varphi^{\prime}, \omega=\omega_{\varphi}, \omega^{\prime}=\omega_{\varphi^{\prime}}$ and $\omega^{\prime \prime}=\omega_{\varphi^{\prime \prime}}$. Then

$$
\begin{aligned}
& -\kappa_{1}\left(\varphi, \varphi^{\prime}\right)-\kappa_{1}\left(\varphi^{\prime}, \varphi^{\prime \prime}\right)+\kappa_{1}\left(\varphi, \varphi^{\prime \prime}\right) \\
& =\int_{M}\left\{-\frac{\log \left(\omega^{\prime n} / \omega^{n}\right)}{2 \pi} \omega^{\prime n}-\frac{\log \left(\omega^{\prime \prime} / \omega^{\prime n}\right)}{2 \pi} \omega^{\prime \prime n}+\frac{\log \left(\omega^{\prime \prime} / \omega^{n}\right)}{2 \pi} \omega^{\prime \prime n}\right\}+ \\
& \int_{M}\left\{\zeta \operatorname{Ric}(\omega) \sum_{i=0}^{n-1} \omega^{n-i-1} \omega^{\prime i}+\zeta^{\prime} \operatorname{Ric}\left(\omega^{\prime}\right) \sum_{i=0}^{n-1} \omega^{\prime n-i-1} \omega^{\prime \prime}-\left(\zeta+\zeta^{\prime}\right) \operatorname{Ric}(\omega) \sum_{i=0}^{n-1} \omega^{n-i-1} \omega^{\prime \prime}\right\} \\
& =\int_{M} \frac{\log \left(\omega^{\prime n} / \omega^{n}\right)}{2 \pi}\left(\omega^{\prime \prime}-\omega^{\prime}\right) \sum_{i=0}^{n-1} \omega^{\prime i} \omega^{\prime \prime n-1-i}+\int_{M} \zeta^{\prime} \sum_{i=0}^{n-1}\left\{\operatorname{Ric}\left(\omega^{\prime}\right) \omega^{\prime n-i-1}-\right. \\
& \left.\operatorname{Ric}(\omega) \omega^{n-i-1}\right\} \omega^{\prime \prime}-\int_{M} \zeta \operatorname{Ric}(\omega) \sum_{i=1}^{n-1} \omega^{n-i-1}\left\{\left(\omega^{\prime \prime}-\omega^{\prime}\right) \sum_{j=0}^{i-1} \omega^{\prime j} \omega^{\prime \prime i-1-j}\right\} .
\end{aligned}
$$

Since $\omega^{\prime \prime}-\omega^{\prime}=\sqrt{-1} \partial \bar{\partial} \zeta^{\prime}$ and $\sqrt{-1} \partial \bar{\partial} \zeta=\omega^{\prime}-\omega$, taking integration by parts, we now obtain (A.2) as follows:

$$
\begin{aligned}
& -\kappa_{1}\left(\varphi, \varphi^{\prime}\right)-\kappa_{1}\left(\varphi^{\prime}, \varphi^{\prime \prime}\right)+\kappa_{1}\left(\varphi, \varphi^{\prime \prime}\right) \\
= & \int_{M} \zeta^{\prime}\left\{\operatorname{Ric}(\omega)-\operatorname{Ric}\left(\omega^{\prime}\right)\right\} \sum_{i=0}^{n-1}{\omega^{\prime}}^{i} \omega^{\prime \prime n-1-i}+\int_{M} \zeta^{\prime} \sum_{i=0}^{n-1}\left\{\operatorname{Ric}\left(\omega^{\prime}\right) \omega^{\prime n-i-1}-\right. \\
& \left.\operatorname{Ric}(\omega) \omega^{n-i-1}\right\} \omega^{\prime \prime}{ }^{i}-\int_{M}\left\{\zeta^{\prime}\left(\omega^{\prime}-\omega\right) \operatorname{Ric}(\omega) \sum_{i=1}^{n-1} \omega^{n-i-1} \sum_{j=0}^{i-1} \omega^{\prime j} \omega^{\prime \prime i-1-j}\right\} \\
= & \int_{M} \zeta^{\prime} \operatorname{Ric}(\omega)\left\{\sum_{i=0}^{n-1}\left({\omega^{\prime}}^{i} \omega^{\prime \prime \prime-1-i}-\omega^{n-i-1} \omega^{\prime \prime}\right)+\sum_{i=1}^{n-1} \sum_{j=0}^{i-1}\left(\omega-\omega^{\prime}\right) \omega^{n-i-1} \omega^{\prime j} \omega^{\prime \prime-1-j}\right\} \\
= & 0 .
\end{aligned}
$$


Similarly, we obtain (A.3) as follows:

$$
\begin{aligned}
& -\kappa_{2}\left(\varphi, \varphi^{\prime}\right)-\kappa_{2}\left(\varphi^{\prime}, \varphi^{\prime \prime}\right)+\kappa_{2}\left(\varphi, \varphi^{\prime \prime}\right) \\
= & \int_{M}\left\{-\zeta \sum_{i=0}^{n} \omega^{n-i}{\omega^{\prime}}^{i}-\zeta^{\prime} \sum_{i=0}^{n}{\omega^{\prime}}^{n-i} \omega^{\prime \prime i}+\left(\zeta+\zeta^{\prime}\right) \sum_{i=0}^{n} \omega^{n-i} \omega^{\prime \prime}{ }^{i}\right\} \\
= & \int_{M}\left\{\zeta \sum_{i=1}^{n} \omega^{n-i}\left(\omega^{\prime \prime}-\omega^{\prime}\right) \sum_{j=0}^{i-1}{\omega^{\prime}}^{i-j-1} \omega^{\prime \prime}-\zeta^{\prime} \sum_{i=1}^{n} \omega^{\prime \prime n-i}\left(\omega^{\prime}-\omega\right) \sum_{j=1}^{i} \omega^{j-1} \omega^{\prime i-j}\right\} \\
= & \int_{M} \zeta^{\prime}\left(\omega^{\prime}-\omega\right)\left(\sum_{i=1}^{n} \sum_{j=0}^{i-1} \omega^{n-i}{\omega^{\prime}}^{i-j-1} \omega^{\prime \prime} j-\sum_{i=1}^{n} \sum_{j=1}^{i} \omega^{j-1} \omega^{\prime i-j} \omega^{\prime \prime n-i}\right)=0 .
\end{aligned}
$$

Let $\left\{\varphi_{t} ; a \leq t \leq b\right\}$ be an arbitrary smooth path in $\tilde{\mathcal{S}}_{\mathbb{C}}$. Along the corresponding path in $\mathcal{S}_{\mathbb{C}}$, we differentiate the identity defining $\kappa\left(\omega, \omega^{\prime}\right)$. In view of Lemma A.1, we obtain

$$
\frac{\partial}{\partial t} \kappa\left(\omega_{0}, \omega_{\varphi_{t}}\right)=\left.\frac{\partial}{\partial \epsilon}\right|_{\epsilon=0} \kappa\left(\omega_{\varphi_{t}}, \omega_{\varphi_{t+\epsilon}}\right)=c_{0}^{-1} \int_{M} \dot{\varphi}_{t}\left\{\sigma\left(\omega_{\varphi_{t}}\right)-n c\right\} \omega_{\varphi_{t}}^{n}
$$

where $\dot{\varphi}_{t}=(d / d t) \varphi_{t}$. Hence, the functional $\mathcal{S}_{\mathbb{C}} \ni \omega \mapsto \kappa\left(\omega_{0}, \omega\right) \in \mathbb{C}$ is a symplectic analogue of the K-energy map. Obviously, $\omega \in \mathcal{S}_{\mathbb{C}}$ is a critical point of the functional if and only if the scalar curvature $\sigma(\omega)$ is constant on $M$.

REMARK A.5. For $\omega, \omega^{\prime} \in \mathcal{S}_{\mathbb{C}}$, we put $\chi\left(\omega, \omega^{\prime}\right):=e^{2 \pi \kappa\left(\omega, \omega^{\prime}\right)}$. Then $\chi: \mathcal{S}_{\mathbb{C}} \times \mathcal{S}_{\mathbb{C}} \rightarrow$ $\mathbb{C}^{*}$ is a functional satisfying the 1-cocycle condition multiplicatively, i.e., for all $\omega, \omega^{\prime}$, $\omega^{\prime \prime} \in \mathcal{S}_{\mathbb{C}}$

(a) $\chi(\omega, \omega)=1$;

(b) $\chi\left(\omega, \omega^{\prime}\right) \chi\left(\omega^{\prime}, \omega^{\prime \prime}\right)=\chi\left(\omega, \omega^{\prime \prime}\right)$.

Let us now assume that $\omega_{0}$ above is a real form, and let $\mathcal{S}$ denote the set of all real forms in $\mathcal{S}_{\mathbb{C}}$. Then it coincides with the definition of $\mathcal{S}$ in Section 5. Take a smooth path $\left\{\varphi_{t} ; 0 \leq t \leq 1\right\}$ in $\mathcal{S}$, and put

$$
\omega=\omega_{\varphi_{0}}, \quad \omega^{\prime}=\omega_{\varphi_{1}}, \quad \zeta=\varphi_{1}-\varphi_{0}
$$

and hence $\omega^{\prime}=\omega+(\sqrt{-1} / 2 \pi) \partial \bar{\partial} \zeta$. In Kähler cases, the following formal computation 
allows Bando to obtain the explicit formula for the K-energy map:

$$
\begin{aligned}
& \sqrt{-1} \partial \bar{\partial} \int_{0}^{1}-\dot{\varphi}_{t}\left\{\sigma\left(\omega_{\varphi_{t}}\right)-n c\right\} \omega_{\varphi_{t}}^{n} d t=\sqrt{-1} \partial \bar{\partial} \int_{0}^{1}-n \dot{\varphi}_{t}\left\{\operatorname{Ric}\left(\omega_{\varphi_{t}}\right)-c \omega_{\varphi_{t}}\right\} \omega_{\varphi_{t}}^{n-1} d t \\
& =\int_{0}^{1}-\left\{\operatorname{Ric}\left(\omega_{\varphi_{t}}\right)-c \omega_{\varphi_{t}}\right\} \frac{d}{d t} \omega_{\varphi_{t}}^{n} d t=-\int_{0}^{1} \operatorname{Ric}\left(\omega_{\varphi_{t}}\right) \frac{d}{d t} \omega_{\varphi_{t}}^{n} d t+\left[\frac{n c}{n+1} \omega_{\varphi_{t}}^{n+1}\right]_{t=0}^{t=1} \\
& =\left[-\operatorname{Ric}\left(\omega_{\varphi_{t}}\right) \omega_{\varphi_{t}}^{n}\right]_{t=0}^{t=1}+\int_{0}^{1}\left\{\frac{d}{d t} \operatorname{Ric}\left(\omega_{\varphi_{t}}\right)\right\} \omega_{\varphi_{t}}^{n} d t+\left[\frac{n c}{n+1} \omega_{\varphi_{t}}^{n+1}\right]_{t=0}^{t=1} \\
& =\left[-\operatorname{Ric}\left(\omega_{\varphi_{t}}\right) \omega_{\varphi_{t}}^{n}\right]_{t=0}^{t=1}+\sqrt{-1} \partial \bar{\partial} \int_{0}^{1}\left\{\left(\omega_{\varphi_{t}}^{n}\right)^{-1} \frac{d}{d t} \omega_{\varphi_{t}}^{n}\right\} \omega_{\varphi_{t}}^{n} d t+\left[\frac{n c}{n+1} \omega_{\varphi_{t}}^{n+1}\right]_{t=0}^{t=1} \\
& =\left\{-\operatorname{Ric}\left(\omega^{\prime}\right) \omega^{\prime n}+\operatorname{Ric}(\omega) \omega^{n}\right\}+(n+1)^{-1} n c\left(\omega^{\prime n+1}-\omega^{n+1}\right) \\
& =\left\{\operatorname{Ric}(\omega)-\operatorname{Ric}\left(\omega^{\prime}\right)\right\} \omega^{\prime n}-\operatorname{Ric}(\omega)\left(\omega^{\prime n}-\omega^{n}\right)+(n+1)^{-1} n c\left(\omega^{\prime n+1}-\omega^{n+1}\right) \\
& =\left\{\operatorname{Ric}(\omega)-\operatorname{Ric}\left(\omega^{\prime}\right)\right\} \omega^{\prime n}-\left(\omega^{\prime}-\omega\right) \operatorname{Ric}(\omega) \sum_{i=0}^{n-1} \omega^{n-1-i} \omega^{\prime i}+\frac{n c\left(\omega^{\prime}-\omega\right)}{n+1} \sum_{i=0}^{n} \omega^{n-i} \omega^{\prime i} \\
& =\sqrt{-1} \partial \bar{\partial}\left\{\frac{\log \left(\omega^{\prime n} / \omega^{n}\right)}{2 \pi} \omega^{\prime n}-\zeta \operatorname{Ric}(\omega) \sum_{i=0}^{n-1} \omega^{n-1-i} \omega^{\prime i}+\frac{n c \zeta}{n+1} \sum_{i=0}^{n} \omega^{n-i} \omega^{\prime i}\right\}
\end{aligned}
$$

This formal computation, as well as the action of the operator $\sqrt{-1} \partial \bar{\partial}$, should be taken over some 'imaginary' space sitting over $M$, where Bando asked why this kind of formal computation is valid after all. By complexifying the smooth path $\left\{\varphi_{t} ; 0 \leq t \leq 1\right\}$, we shall now give some geometric answer to this in our symplectic situation. By (A.4), this consequently gives another proof of Lemma A.1 not only in Kähler cases, but also in the case where the above $\omega, \omega^{\prime}, \omega^{\prime \prime}$ are pseudo-Kähler forms on $M$ joined together by piecewise-smooth paths of pseudo-Kähler forms in the symplectic class.

(A.6) Justification of the formal computation: For the annulus $D:=\{z \in \mathbb{C} ; 1 \leq$ $|z| \leq e\}$, we define a real-valued smooth function $s=s(z)$ on $D$ by setting $s:=\log |z|$ for each $z \in D$. Then by

$$
S^{1} \times D \rightarrow D, \quad\left(e^{\sqrt{-1} \theta}, z\right) \mapsto e^{\sqrt{-1} \theta} z
$$

the group $S^{1}$ acts on $D$. Put $\varphi_{s, t}:=\varphi_{s t}$ and $\zeta_{s}:=\varphi_{s, 1}-\varphi_{s, 0}=\varphi_{s}-\varphi_{0}$. For $0 \leq s \leq 1$, we further put

$$
\begin{aligned}
& \eta(s):=\frac{\log \left(\omega_{\varphi_{s}}^{n} / \omega^{n}\right)}{2 \pi} \omega_{\varphi_{s}}^{n}-\zeta_{s} \operatorname{Ric}(\omega) \sum_{i=0}^{n-1} \omega^{n-1-i} \omega_{\varphi_{s}}^{i}+\frac{n c \zeta_{s}}{n+1} \sum_{i=0}^{n} \omega^{n-i} \omega_{\varphi_{s}}^{i}, \\
& \tau_{1}(s):=\frac{1}{2 \pi} \int_{0}^{1}\left(\int_{M}-\frac{\partial \varphi_{s, t}}{\partial t}\left\{\sigma\left(\omega_{\varphi_{s, t}}\right)-n c\right\} \omega_{\varphi_{s, t}}^{n}\right) d t
\end{aligned}
$$

and define $\tau_{2}(s):=\int_{M} \eta(s)$. Then $\tau_{1}(s(z))$ and $\tau_{2}(s(z))$ are $S^{1}$-invariant real-valued smooth functions on $D$. Define a real $(1,1)$-form $\tilde{\omega}_{\varphi_{s, t}}$ and $\widetilde{\operatorname{Ric}}\left(\omega_{\varphi_{s, t}}\right)$ on $D \times M$ by

$$
\begin{aligned}
& \tilde{\omega}_{\varphi_{s, t}}=\omega_{0}+(\sqrt{-1} / 2 \pi) \partial \bar{\partial} \varphi_{s, t}, \\
& \widetilde{\operatorname{Ric}}\left(\omega_{\varphi_{s, t}}\right)=(-\sqrt{-1} / 2 \pi) \partial \bar{\partial} \log \omega_{\varphi_{s, t}}^{n}
\end{aligned}
$$


where the operator $\partial \bar{\partial}$ is taken on the complex manifold $D \times M$, and $\tilde{\omega}_{\varphi_{s, t}}$ is distinguished from the realtive $(1,1)$-form $\omega_{\varphi_{s, t}}$ on $D \times M$ over $D$. Define

$$
\tilde{\eta}(s):=\frac{\log \left(\tilde{\omega}_{\varphi_{s, 1}}^{n} / \tilde{\omega}_{\varphi_{s, 0}^{n}}\right)}{2 \pi} \tilde{\omega}_{\varphi_{s, 1}}^{n}-\zeta_{s} \operatorname{Ric}\left(\tilde{\omega}_{\varphi_{s, 0}}\right) \sum_{i=0}^{n-1} \tilde{\omega}_{\varphi_{s, 0}}^{n-1-i} \tilde{\omega}_{\varphi_{s, 1}}^{i}+\frac{n c \zeta_{s}}{n+1} \sum_{i=0}^{n} \tilde{\omega}_{\varphi_{s, 0}}^{n-i} \tilde{\omega}_{\varphi_{s, 1}}^{i},
$$

where we here observe that $\omega_{\varphi_{s, 0}}=\omega$ and $\omega_{\varphi_{s, 1}}=\omega_{\varphi_{s}}$. For every smooth realvalued function $\phi=\phi(z)$ on $D$ whose support is a compact subset of the interior $\{z \in \mathbb{C} ; 1<|z|<e\}$ of $D$, we have (see [23] for a similar computation in a different context):

$$
\begin{aligned}
& \int_{D} \phi(z) \sqrt{-1} \partial \bar{\partial} \tau_{1}(s(z))=\int_{D} \tau_{1}(s(z)) \sqrt{-1} \partial \bar{\partial} \phi(z) \\
& =\frac{1}{2 \pi} \int_{0}^{1}\left\{\int_{D \times M}-n \frac{\partial \varphi_{s, t}}{\partial t}\left\{\widetilde{\operatorname{Ric}}\left(\omega_{\varphi_{s, t}}\right)-c \tilde{\omega}_{\varphi_{s, t}}\right\} \tilde{\omega}_{\varphi_{s, t}^{n-1}}^{n-1} \sqrt{-1} \partial \bar{\partial} \phi(z)\right\} d t \\
& =\frac{1}{2 \pi} \int_{0}^{1}\left\{\int_{D \times M}-n \phi(z) \sqrt{-1} \partial \bar{\partial}\left(\frac{\partial \varphi_{s, t}}{\partial t}\right)\left\{\widetilde{\operatorname{Ric}}\left(\omega_{\varphi_{s, t}}\right)-c \tilde{\omega}_{\varphi_{s, t}}\right\} \tilde{\omega}_{\varphi_{s, t}}^{n-1}\right\} d t \\
& \left.=\int_{D \times M}\left\{\phi(z) \int_{0}^{1}-\widetilde{\operatorname{Ric}}\left(\omega_{\varphi_{s, t}}\right)-c \tilde{\omega}_{\varphi_{s, t}}\right\} \frac{\partial}{\partial t} \tilde{\omega}_{\varphi_{s, t}}^{n}\right\} d t \\
& =\int_{D \times M} \phi(z)\left\{\int_{0}^{1} \widetilde{\operatorname{Ric}}\left(\omega_{\varphi_{s, t}}\right) \frac{\partial}{\partial t} \tilde{\omega}_{\varphi_{s, t}}^{n} d t+\left[\frac{n c}{n+1} \tilde{\omega}_{\varphi_{s, t}}^{n+1}\right]_{t=0}^{t=1}\right\} \\
& =\int_{D \times M} \phi(z)\left\{\left[-\widetilde{\operatorname{Ric}}\left(\omega_{\varphi_{s, t}}\right) \tilde{\omega}_{\varphi_{s, t}}^{n}\right]_{t=0}^{t=1}+\int_{0}^{1}\left\{\frac{\partial}{\partial t} \widetilde{\operatorname{Ric}}\left(\omega_{\varphi_{s, t}}\right)\right\} \tilde{\omega}_{\varphi_{s, t}}^{n} d t+\left[\frac{n c}{n+1} \tilde{\omega}_{\varphi_{s, t}}^{n+1}\right]_{t=0}^{t=1}\right\},
\end{aligned}
$$

where in the last line, we have

$$
\begin{aligned}
& \int_{D \times M} \phi(z) \int_{0}^{1}\left\{\frac{\partial}{\partial t} \widetilde{\operatorname{Ric}}\left(\omega_{\varphi_{s, t}}\right)\right\} \tilde{\omega}_{\varphi_{s, t}}^{n} d t \\
= & -\int_{D \times M} \phi(z) \int_{0}^{1} \sqrt{-1} \partial \bar{\partial}\left\{\left(\omega_{\varphi_{s, t}}^{n}\right)^{-1} \frac{\partial}{\partial t} \omega_{\varphi_{s, t}}^{n}\right\} \tilde{\omega}_{\varphi_{s, t}}^{n} d t \\
= & -\int_{D \times M} \sqrt{-1} \partial \bar{\partial} \phi(z) \int_{0}^{1}\left\{\left(\omega_{\varphi_{s, t}}^{n}\right)^{-1} \frac{\partial}{\partial t} \omega_{\varphi_{s, t}}^{n}\right\} \tilde{\omega}_{\varphi_{s, t}}^{n} d t \\
= & -\int_{D \times M} \sqrt{-1} \partial \bar{\partial} \phi(z) \int_{0}^{1}\left\{\left(\omega_{\varphi_{s, t}}^{n}\right)^{-1} \frac{\partial}{\partial t} \omega_{\varphi_{s, t}}^{n}\right\} \omega_{\varphi_{s, t}}^{n} d t \\
= & -\int_{D \times M} \sqrt{-1} \partial \bar{\partial} \phi(z)\left[\omega_{\varphi_{s, t}}^{n}\right]_{t=0}^{t=1}=-\int_{D \times M} \sqrt{-1} \phi(z) \partial \bar{\partial}\left[\omega_{\varphi_{s, t}}^{n}\right]_{t=0}^{t=1}=0 .
\end{aligned}
$$

Hence, in view of the original formal computation above, we obtain

$$
\begin{aligned}
& \int_{D} \phi(z) \sqrt{-1} \partial \bar{\partial} \tau_{1}(s(z))=\int_{D \times M} \phi(z)\left\{\left[-\widetilde{\operatorname{Ric}}\left(\omega_{\varphi_{s, t}}\right) \tilde{\omega}_{\varphi_{s, t}}^{n}\right]_{t=0}^{t=1}+\left[\frac{n c}{n+1} \tilde{\omega}_{\varphi_{s, t}}^{n+1}\right]_{t=0}^{t=1}\right\} \\
& =\int_{D \times M} \phi(z)\left\{-\widetilde{\operatorname{Ric}}\left(\omega_{\varphi_{s, 1}}\right) \tilde{\omega}_{\varphi_{s, 1}}^{n}+\widetilde{\operatorname{Ric}}\left(\omega_{\varphi_{s, 0}}\right) \tilde{\omega}_{\varphi_{s, 0}}^{n}+(n+1)^{-1} n c\left(\tilde{\omega}_{\varphi_{s, 1}}^{n+1}-\tilde{\omega}_{\varphi_{s, 0}}^{n+1}\right)\right\} \\
& =\int_{D \times M} \phi(z) \sqrt{-1} \partial \bar{\partial} \tilde{\eta}(s)=\int_{D \times M}\{\sqrt{-1} \partial \bar{\partial} \phi(z)\} \tilde{\eta}(s)=\int_{D \times M}\{\sqrt{-1} \partial \bar{\partial} \phi(z)\} \eta(s) \\
& =\int_{D}\{\sqrt{-1} \partial \bar{\partial} \phi(z)\} \tau_{2}(s)=\int_{D} \phi(z) \sqrt{-1} \partial \bar{\partial} \tau_{2}(s(z)) .
\end{aligned}
$$


Therefore, $\partial \bar{\partial}\left\{\tau_{2}(s(z))-\tau_{1}(s(z))\right\}=0$ on $D$. Since the function $\tau_{2}(s(z))-\tau_{1}(s(z))$ on the annulus $D$ is $S^{1}$-invariant, it then follows that

$$
\frac{\partial^{2}}{\partial s^{2}}\left\{\tau_{2}(s)-\tau_{1}(s)\right\}=0, \quad 0 \leq s \leq 1 .
$$

On the other hand, the equality $\tau_{1}(0)=0=\tau_{2}(0)$ obviously holds. Furthermore, by the same computation as in (A.4), we have $\dot{\tau}_{1}(0)=\dot{\tau}_{2}(0)$. Hence, $\tau_{1}(s)=\tau_{2}(s)$ for all $0 \leq s \leq 1$. Thus $\tau_{1}(1)=\tau_{2}(1)$, as required.

\section{REFERENCES}

[1] M.F. AтіYaH, Convexity and commuting hamiltonians, Bull. Londn Math. Soc., 14 (1982), pp. $1-15$.

[2] S. BANDo, Existence problem of Einstein-Kähler metrics - positive scalar curvature case, (Japanese) Sûgaku, 50 (1998), pp. 358-367.

[3] N. BERLINe ET M. Vergne, Zeros d'un champ de vecteurs et classes characteristiques equivariantes, Duke Math. J., 50 (1983), pp. 539-549.

[4] T. Delzant, Hamiltoniens périodiques et image convexe de l'application moment, Bull. Soc. Math. France, 116 (1988), pp. 315-339.

[5] J.J. DuistermaAt And G.D. HeCKman, On the variation in the cohomology of the symplectic form of the reduced phase space, Invent. Math., 69 (1982), pp. 259-268; Addendum, Invent. Math., 72 (1983), pp. 153-158.

[6] A. FUנIKI, On automorphism groups of compact Kähler manifolds, Invent. Math., 44 (1978), pp. $225-258$.

[7] A. FUJIKI, Kähler quotient and equivariant cohomology, in "Moduli of vector bundles" (M. Maruyama, ed.), Marcel Dekker, New York-Basel-Hong Kong, 1996.

[8] A. Futaki, An obstruction to the existence of Einstein Kähler metrics, Invent. Math., 73 (1983), pp. 437-443.

[9] A. FUTAKI, Kähler-Einstein metrics and integral invariants, Lecture Notes in Math., 1314 (1988), Springer-Verlag, Heidelberg-New York-Paris-Tokyo.

[10] A. FUtAKI AND T. MABUCHI, Bilinear forms and extremal Kähler vector fields associated with Kähler classes, Math. Ann., 301 (1995), pp. 199-210.

[11] A. FUTAKI AND S. MORITA, Invariant polynomials of the automophism group of a compact complex manifold, J. Diff. Geom., 21 (1985), pp. 135-142.

[12] A. Futaki AND Y. NAKAGAWA, Characters of automorphism groups associated with Kähler classes and functionals with cocycle conditions, Kodai Math. J., 24 (2001), pp. 1-14.

[13] R.E. GompF, A new construction of symplectic manifolds, Annals of Math., 142 (1995), pp. 527-595.

[14] V. Guillemin and S. Sternberg, Convexity properties of the momentum mapping, Invent. Math., 67 (1982), pp. 491-513.

[15] V. Guillemin And S. Sternberg, Geometric quantization and multiplicities of group representation, Invent. Math., 67 (1982), pp. 515-538.

[16] A.D. Hwang, On existence of Kähler metrics with constant scalar curvature, Osaka J. Math., 31 (1994), pp. 561-595.

[17] F. C. KIRWAN, Cohomology of quotients in symplectic and algebraic geometry, Princeton University Press, New Jersey, (1984).

[18] S. KOBAYASHI, Transformation groups in differential geometry, Springer Verlag, BerlinHeidelberg-New York, 1972.

[19] T. MaBuCHI, K-energy maps integrating Futaki invariants, Tôhoku Math. J., 38 (1986), pp. $575-593$.

[20] T. MABUCHI, $\mathbb{Q}$-rationality of moment maps, Proc. Japan Acad., 69 (1993), Ser. A, pp. 392-394.

[21] K. ONo, Equivariant projectve imbedding theorem for symplectic manifolds, J. Fac. Sci. Univ. Tokyo, Sect. IA, Math., 35 (1988), pp. 381-392.

[22] H. NAKaGaWA, Bando-Calabi-Futaki character of Kähler orbifolds, Math. Ann., 314 (1999), pp. 369-380.

[23] G. TIAn, Kähler-Einstein metrics with positive scalar curvature, Invent. Math., 130 (1997), pp. $1-37$. 
